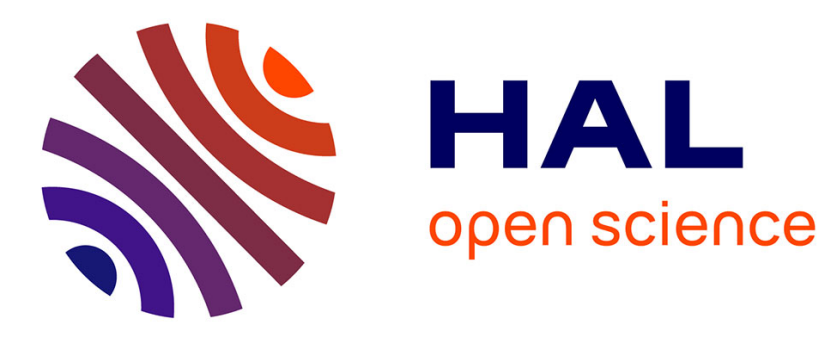

\title{
Identification of high-dimension polynomial chaos expansions with random coefficients for non-Gaussian tensor-valued random fields using partial and limited experimental data
}

Christian Soize

\section{To cite this version:}

Christian Soize. Identification of high-dimension polynomial chaos expansions with random coefficients for non-Gaussian tensor-valued random fields using partial and limited experimental data. Computer Methods in Applied Mechanics and Engineering, 2010, 199 (33-36), pp.2150-2164. 10.1016/j.cma.2010.03.013 . hal-00684324

\section{HAL Id: hal-00684324 \\ https://hal.science/hal-00684324}

Submitted on 1 Apr 2012

HAL is a multi-disciplinary open access archive for the deposit and dissemination of scientific research documents, whether they are published or not. The documents may come from teaching and research institutions in France or abroad, or from public or private research centers.
L'archive ouverte pluridisciplinaire HAL, est destinée au dépôt et à la diffusion de documents scientifiques de niveau recherche, publiés ou non, émanant des établissements d'enseignement et de recherche français ou étrangers, des laboratoires publics ou privés. 


\title{
Identification of high-dimension polynomial chaos expansions with random coefficients for non-Gaussian tensor-valued random fields using partial and limited experimental data
}

\author{
C. Soize* \\ Université Paris-Est, Laboratoire Modélisation et Simulation Multi-Echelle, MSME UMR 8208 \\ CNRS, 5 bd Descartes, 77454 Marne-la-Vallée Cedex 2, France
}

\begin{abstract}
This paper is devoted to the identification of high-dimension polynomial chaos expansions with random coefficients for non-Gaussian tensor-valued random fields using partial and limited experimental data. The experimental data sets correspond to partial experimental data made up of an observation vector which is the response of a stochastic boundary value problem depending on the tensor-valued random field which has to be identified. So an inverse stochastic problem has to be solved to carry out the identification of the random field. A complete methodology is proposed to solve this challenging problem and consists in introducing a family of prior probability models, in identifying an optimal prior model in the constructed family using the experimental data, in constructing a statistical reduced order optimal prior model, in constructing the polynomial chaos expansion with deterministic vector-valued coefficients of the reduced order optimal prior model and finally, in constructing the probability distribution of random coefficients of the polynomial chaos expansion and in identifying the parameters using experimental data. An application is presented for which several millions of random coefficients are identified solving an inverse stochastic problem.
\end{abstract}

Key words: Polynomial chaos expansion, identification, inverse stochastic problem, random field, stochastic process, stochastic boundary value problem.

\footnotetext{
${ }^{*}$ Corresponding author

Email address: christian.soize@univ-paris-est.fr(C. Soize)
} 


\section{Introduction}

The methodology to construct a polynomial chaos expansion of random fields has been introduced in [21, 20, 58]) (and is analyzed in [55, 62] for an arbitrary measure). The polynomial chaos expansions of stochastic processes and random fields, and application to stochastic boundary value problems, have generated many works in the last decade (see $[8,11,12,13,14,15,17,18,19,22$, $23,24,27,28,29,30,31,32,33,37,38,39,40,42,43,44,45,46,47,52$, $53,63,66,67,68,69])$. The stochastic inverse methods and the Bayesian inference approach to inverse problems have recently received a particular attention (see $[34,35,36,64,65,70]$ ). The problem relative to the identification with experimental data of the deterministic coefficients of the chaos expansion of a non-Gaussian real-valued random field using the maximum likelihood has been introduced in [9] and more recently, has been used in [59] and revisited in [6]. In practice, the identification of these deterministic coefficients is performed using a finite length of experimental data sets. Consequently, the maximum likelihood statistical estimator of these coefficients is not completely convergent and therefore, there are residual statistical fluctuations which can be modeled in representing the deterministic coefficients by random coefficients. Recently, an interesting work [7] proposes to construct the probability model of these random coefficients by using the asymptotic sampling Gaussian distribution constructed with the Fisher information matrix and which is a consistent and asymptotically efficient estimator. Such an approach has been used for model validation [16, 41]. Very recently, in [1], as a continuation of [56], the identification of Bayesian posteriors for the coefficients of chaos expansions is proposed. In this approach, the usual deterministic coefficients of the chaos expansion are replaced by random coefficients in order to quantify the uncertainties induced by the errors. Two types of errors are considered. The first type is due to the use of a relatively low order of the maximum degree of polynomials in the chaos expansion. The second type is due to the finite length of the experimental data set. First, a prior probability density function of the random coefficients is constructed by using the maximum likelihood method and the projection of the experimental data sets on the eigenvectors of the Karhunen-Loeve decomposition. A parametric representation of the posterior probability density function is constructed using the formalism introduced in [56] for the reduced chaos decomposition with random coefficients of vectorvalued random variables. Then the parameters of the posterior probability density function are updated by the Bayes method with the experimental data set. 
This paper is devoted to the identification of high-dimension polynomial chaos expansion with random coefficients for non-Gaussian tensor-valued random fields using partial and limited experimental data. The main hypotheses are thus:

- a non-Gaussian tensor-valued random field must be identified, and not a realvalued random field.

- the experimental data sets which are available do not correspond to direct field measurements by image processing of the random field to be identified but correspond to partial experimental data made up of an observation vector which is the response of a stochastic boundary value problem depending on the tensor-valued random field which has to be identified. So an inverse stochastic problem has to be solved to carry out the identification of the random field.

- the convergence of the chaos expansion is generally not analyzed due to the difficulties to solve the inverse stochastic problem which can be in very high dimension, that is to say which can have a very large number of coefficients (several millions in the chaos expansion) which have to be identified In this paper we propose to carry out such an analysis.

Below, we define the challenging problem which has to be solved and we show the main difficulties induced by this problem.

(1) Stochastic boundary value problem. We consider a boundary value problem for a vector-valued field $\left\{\boldsymbol{u}(\boldsymbol{x})=\left(u_{1}(\boldsymbol{x}), u_{2}(\boldsymbol{x}), u_{3}(\boldsymbol{x})\right), \boldsymbol{x} \in \Omega\right\}$ on an open bounded domain $\Omega$ of $\mathbb{R}^{3}$ with generic point $\boldsymbol{x}=\left(x_{1}, x_{2}, x_{3}\right)$. This boundary value problem depends on a non-Gaussian fourth-order tensor-valued random field $\{\boldsymbol{C}(\boldsymbol{x}), \boldsymbol{x} \in \Omega\}$ in which $\boldsymbol{C}(\boldsymbol{x})=\left\{\boldsymbol{C}_{i j k \ell}(\boldsymbol{x})\right\}_{i j k \ell}$, which is unknown and which has to be identified solving an inverse stochastic problem. The boundary $\partial \Omega$ of domain $\Omega$ is written as $\Gamma_{0} \cup \Gamma_{\text {obs }} \cup \Gamma$. Field $\boldsymbol{u}$ is only observed on $\Gamma_{\text {obs }}$ which means that the system is partially observable.

(2) Stochastic finite element approximation of the stochastic boundary value problem. The above stochastic boundary value problem is discretized by the finite element method. Let $\mathcal{I}=\left\{\boldsymbol{x}^{1}, \ldots, \boldsymbol{x}^{N_{p}}\right\} \subset \Omega$ be the finite subset of $\Omega$ made up of all the integrations points of the finite elements of the mesh of $\Omega$. For all $x$ fixed in $\mathcal{I} \subset \Omega$, the fourth-order tensor-valued random variable $\boldsymbol{C}(\boldsymbol{x})$ is represented by a real random matrix $[\boldsymbol{A}(\boldsymbol{x})]$ such that $[\boldsymbol{A}(\boldsymbol{x})]_{I J}=\boldsymbol{C}_{i j k \ell}(\boldsymbol{x})$ with a given adapted correspondence $I=(i, j)$ and $J=(k, \ell)$. It should be noted that mathematical properties on the matrix-valued random field $\{[\boldsymbol{A}(\boldsymbol{x})], \boldsymbol{x} \in \Omega\}$ are necessary in order to preserve the mathematical properties of the boundary value problem.

Let $\boldsymbol{U}=\left(U_{1}, \ldots, U_{m}\right)$ be the $\mathbb{R}^{m}$-valued random vector of the observed de- 
grees of freedom (DOF) corresponding to the finite element approximation of the trace on $\Gamma_{\text {obs }}$ of random field $\boldsymbol{u}$. Vector $\boldsymbol{U}$ will be called the observation vector. Consequently, the random observation vector $\boldsymbol{U}$ appears as the unique deterministic nonlinear transformation of the finite family of $N_{p}$ dependent real random matrices $\{[\boldsymbol{A}(\boldsymbol{x})], \boldsymbol{x} \in \mathcal{I}\}$. This set of random matrices can then be represented by

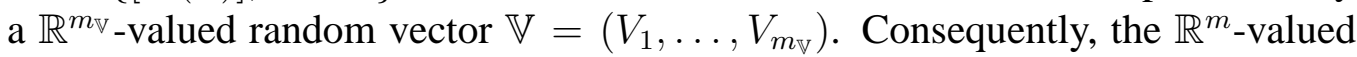
random vector $\boldsymbol{U}$ of the observation vector can be written as

$$
\boldsymbol{U}=\boldsymbol{h}(\mathbb{V})
$$

in which $v \mapsto \boldsymbol{h}(v)$ is a deterministic nonlinear transformation from $\mathbb{R}^{m_{\mathbb{V}}}$ into $\mathbb{R}^{m}$ which can be constructed solving the discretized boundary value problem.

(3) Experimental data sets. It is assumed that $\nu_{\text {exp }}$ experimental data are available for the observation vector. Each experimental data set corresponds to partial experimental data (only the trace of the displacement field on $\Gamma_{\text {obs }}$ is observed) with a limited length ( $\nu_{\text {exp }}$ is small). These $\nu_{\text {exp }}$ experimental data sets correspond to measurements for $\nu_{\text {exp }}$ experimental configurations corresponding to the same boundary value problem. For configuration $\ell$, with $\ell=1, \ldots, \nu_{\text {exp }}$, the observation vector corresponding to $\boldsymbol{U}$ for the computational model is denoted by $\boldsymbol{u}^{\exp , \ell}$ and belongs to $\mathbb{R}^{m}$. Therefore, the available data are made up of the $\nu_{\exp }$ vectors $\boldsymbol{u}^{\exp , 1}, \ldots, \boldsymbol{u}^{\exp , \nu_{\exp }}$ in $\mathbb{R}^{m}$. Below, it is assumed that $\boldsymbol{u}^{\exp , 1}, \ldots, \boldsymbol{u}^{\exp , \nu_{\exp }}$ can be used as $\nu_{\text {exp }}$ independent realizations of a random vector $\boldsymbol{U}^{\exp }$ corresponding to random vector $\boldsymbol{U}$ but random vectors $\boldsymbol{U}^{\exp }$ and $\boldsymbol{U}$ are not defined on the same probability space.

(4) Construction of the polynomial chaos expansion with random coefficients for the non-Gaussian matrix-valued random field. As explained in point (2) above, using the finite element discretization, the non-Gaussian matrix-valued random field $\{[\boldsymbol{A}(\boldsymbol{x})], \boldsymbol{x} \in \Omega\}$ is represented by the $\mathbb{R}^{m_{\mathbb{V}}}$-valued random vector $\mathbb{V}$. Let $\{[\underline{A}(\boldsymbol{x})], \boldsymbol{x} \in \Omega\}$ with $[\underline{A}(\boldsymbol{x})]=E\{[\boldsymbol{A}(\boldsymbol{x})]\}$ be the matrix-valued mean function in which $E$ is the mathematical expectation. The mean value $\underline{\mathbb{V}}=E\{\mathbb{V}\}$ is then directly deduced from the matrix-valued mean function $\{[\underline{A}(\boldsymbol{x})], \boldsymbol{x} \in \Omega\}$ which coincides with the value of the deterministic nominal model (the mean model). In this paper, it is assumed that $[\underline{A}(\boldsymbol{x})]=[\underline{A}]$ is independent of $\boldsymbol{x}$. This implies that vector $\underline{\mathbb{V}}$ depends only on the real matrix $[\underline{A}]$ which has a small dimension. It should be noted that such an assumption can easily be removed without changing the methodology proposed. 
In this paper, we are interested (a) in constructing a polynomial chaos expansion with random coefficients of the non-Gaussian random vector $\mathbb{V}$ and (b) in identifying the random coefficients of this chaos expansion using a limited number $\nu_{\text {exp }}$ of experimental data sets, each data set corresponding to partial observations of the system. We then have to solve an inverse stochastic problem.

The methodology to construct a polynomial chaos expansion of random fields (see $[21,20,58]$ ) consists (a) in reducing the field with a Karhunen-Loeve (KL) expansion and then (b) in performing the polynomial chaos expansion of the KL expansion random coordinates. Presently, because the tensor-valued random field to be identified is unknown, in order to be able to represent any second-order random field, the Gaussian space is required and consequently, the Gaussian chaos decomposition is used. For the spatially sampled field, the first stage means that random vector $\mathbb{V}$ (which is assumed to be a second-order random variable) is written (finite approximation of the Karhunen-Loeve expansion for the matrixvalued random field $[\boldsymbol{A}]$ ) as

$$
\mathbb{V} \simeq \underline{\mathbb{V}}+\sum_{j=1}^{n} \sqrt{\lambda_{j}} \eta_{j} \mathbb{W}^{j}
$$

in which symbol " $\simeq$ " means that the right-hand side in Eq. (2) is an approximation of $\mathbb{V}$ for which mean-square convergence is reached for $n$ sufficiently large.

The vectors $\mathbb{W}^{1}, \ldots, \mathbb{W}^{n}$ constitute an orthonormal family in $\mathbb{R}^{m}$ (for the Euclidean inner product) made up of the eigenvectors associated with the first largest positive eigenvalues $\lambda_{1} \geq \ldots \geq \lambda_{n}$ of the covariance matrix $\left[C_{\mathbb{V}}\right]$ of the random vector $\mathbb{V}$. The $\mathbb{R}^{n}$-valued second-order centered random variable $\boldsymbol{\eta}=\left(\eta_{1}, \ldots, \eta_{n}\right)$ is such that

$$
E\{\boldsymbol{\eta}\}=\mathbf{0} \quad, \quad E\left\{\boldsymbol{\eta} \boldsymbol{\eta}^{T}\right\}=\left[I_{n}\right],
$$

in which $\left[I_{n}\right]$ is the $(n \times n)$ identity matrix. Note that the components of the nonGaussian random variable $\boldsymbol{\eta}$ are not correlated but are statistically dependent. The polynomial Chaos expansion with deterministic coefficients of random vector $\boldsymbol{\eta}$ is written as

$$
\boldsymbol{\eta} \simeq \sum_{\alpha=1}^{N} \boldsymbol{y}^{\alpha} \Psi_{\alpha}(\boldsymbol{\Xi})
$$

in which the coefficients $\boldsymbol{y}^{1}, \ldots, \boldsymbol{y}^{N}$ are vectors in $\mathbb{R}^{n}$ such that

$$
\sum_{\alpha=1}^{N} \boldsymbol{y}^{\alpha} \boldsymbol{y}^{\alpha T}=\left[I_{n}\right]
$$


The real valued random variables $\Psi_{1}(\boldsymbol{\Xi}), \ldots, \Psi_{N}(\boldsymbol{\Xi})$ are the renumbered normalized Hermite polynomials of the $\mathbb{R}^{N_{g}}$-valued normalized Gaussian random variable $\boldsymbol{\Xi}=\left(\Xi_{1}, \ldots, \Xi_{N_{g}}\right)$ (that is to say $E\{\boldsymbol{\Xi}\}=\mathbf{0}$ and $E\left\{\boldsymbol{\Xi} \boldsymbol{\Xi}^{T}\right\}=\left[I_{N_{g}}\right]$ ) such that for all $\alpha$ and $\beta$ in $\{1, \ldots, N\}$,

$$
E\left\{\Psi_{\alpha}(\boldsymbol{\Xi})\right\}=0 \quad, \quad E\left\{\Psi_{\alpha}(\boldsymbol{\Xi}) \Psi_{\beta}(\boldsymbol{\Xi})\right\}=\delta_{\alpha \beta} \quad,
$$

in which $\delta_{\alpha \beta}$ is the Kronecker symbol. It should be noted that the constant Hermite polynomial with index $\alpha=0$ is not included in Eq. (4). If $N_{d}$ is the integer number representing the maximum degree of the Hermite polynomials in Eq. (4), then the number $N$ of chaos in Eq. (4) is $N=h\left(N_{g}, N_{d}\right)=\left(N_{d}+N_{g}\right) ! /\left(N_{d} ! N_{g} !\right)-1$. In Eq. (4), symbol " $\simeq$ " means that the right-hand side is an approximation of $\boldsymbol{\eta}$ for which mean-square convergence is reached for $N_{d}$ and $N_{g}$ sufficiently large. From Eqs. (2) and (4), it can be deduced the polynomial chaos expansion of random vector $\mathbb{V}$,

$$
\mathbb{V} \simeq \underline{\mathbb{V}}+\sum_{\alpha=1}^{N} \boldsymbol{v}^{\alpha} \Psi_{\alpha}(\boldsymbol{\Xi}) \quad, \quad \boldsymbol{v}^{\alpha}=\sum_{j=1}^{n} \sqrt{\lambda_{j}} y_{j}^{\alpha} \mathbb{W}^{j}
$$

in which $\left\{\boldsymbol{v}^{1}, \ldots, \boldsymbol{v}^{N}\right\}$ is a family of $N$ vectors in $\mathbb{R}^{m}$ which are not orthogonal.

(5) Difficulties concerning the identification of the polynomial chaos expansion in high dimension solving an inverse stochastic problem. This challenging problem of identification is due to the presence of the following major difficulties.

(d1) The first one is due to the fact that the experimental data which are available do not correspond to direct field measurements by image processing in domain $\Omega$ allowing realizations of $\mathbb{V}$ to be directly deduced but correspond to partial experimental data made up of observation vector $\boldsymbol{U}$. So an inverse stochastic problem has to be solved to carry out the identification of the coefficients.

(d2) The second difficulty is related to the size of the inverse problem which has to be solved. The identification of the chaos expansion has to be converged with respect to $N$ for problems for which dimension $n$ of the reduced model can be large. This means that the number of real coefficients $y_{j}^{\alpha}$ which has to be identified is $n \times N$ (for instance, for $N=15000$ and $n=500$, there are 7500000 real coefficients which have to be identified).

(d3) The third one is related to the length of the experimental data which are effectively available. If the number of experimental data is less than the number of coefficients which have to be identified, we have an ill-posed problem (if no additional information to experimental data is available). 
(d4) The covariance matrix $\left[C_{\mathbb{V}}\right]$ of random vector $\mathbb{V}$ must be known to construct the representation defined by Eq. (2). In the context of a statistical identification, this means that a lot of experimental data relative to the complete displacement random field $\{\boldsymbol{u}(\boldsymbol{x}), \boldsymbol{x} \in \Omega\}$ should be available with an adapted spatial resolution at the meso-scale. From point (d3) above, clearly the assumptions do not allow the covariance matrix $\left[C_{\mathbb{V}}\right]$ of $\mathbb{V}$ to be estimated from the experimental data. It should be noted that if the basis made up of the eigenvectors $\mathbb{W}^{1}, \ldots, \mathbb{W}^{n}$ of $\left[C_{\mathbb{V}}\right]$ was replaced by another orthogonal basis, then the components of $\boldsymbol{\eta}$ would become correlated and above all, the mean-square convergence of the right-hand side of Eq. (2) would be reached for a value of $n$ much more larger and consequently, the statistical reduction would not be efficient. In such a case, firstly $n$ can be so large that the identification problem to be performed becomes unrealistic but secondly, the value of $n$ is unknown because only partial experimental data are available.

(d5) An uncertainty model induced by model errors and sampling errors should be introduced to improve the robustness of the identified representation.

In this paper, we present a methodology and we validate it to solve the challenging problem of the identification in high dimension of the polynomial chaos expansion of random field with partial and limited experimental data. The method proposed is efficient in high dimension, that is to say, for which $\nu_{\text {exp }}$ is small, $m_{\mathbb{V}}$, $n, N_{d}, N_{g}$ and $N$ can be high or very high. For instance, for the application shown in Section 4, the number of sets of partial experimental data is $\nu_{\exp }=110$. The dimension of random vector $\mathbb{V}$ is $m_{\mathbb{V}}=36288$. The dimension of random vector $\boldsymbol{\eta}$ in the expansion of $\mathbb{V}$ is $n=550$. The maximum degree of the Hermite polynomials is $N_{d}=22$ and the dimension of the vector-valued Gaussian germ is $N_{g}=4$ yielding $N=14949$ chaos. Such a relatively large dimension $n=550$ of the Karhunen-Loeve expansion of the random field is encountered if the spatial correlation length of the random field is smaller than the size of the domain and when a good convergence of the expansion is searched. This is the case for mesoscale stochastic modeling of complex microstructures such as the microstructures of live tissues. For instance, in Section 4, the value $n=550$ corresponds to a correlation length 0.3 which has to be compared to the size of the domain which is 1 and the convergence is reached with a relative error of 0.1 .

In Section 2, each step of the methodology is developed. The fundamental aspects of the algorithms are presented in Section 3. The last section deals with an application in high dimension. 


\section{Methodology}

In this section, we summarize the methodology proposed to solve the challenging problem introduced in Section 1 and then we develop it.

\subsection{Summarizing the methodology}

The methodology proposed is made up of five main steps.

Step 1. Introduction of a family of prior models $\mathbb{V}^{\text {prior }}(\boldsymbol{w})$ for random vector $\mathbb{V}$ depending on a vector-valued parameter $\boldsymbol{w}$ with a very low dimension. Typically, the components of $\boldsymbol{w}$ will be the algebraically independent parameters of matrix $[\underline{A}]$ (which generates the mean value $\underline{\mathbb{V}}$ ), the spatial correlation lengths and the dispersion parameters controlling the statistical fluctuations of matrix-valued random field $[\boldsymbol{A}]$.

Step 2. Use of the experimental data $\left\{\boldsymbol{u}^{\exp , 1}, \ldots, \boldsymbol{u}^{\exp , \nu_{\exp }}\right\}$ to identify the optimal value $\boldsymbol{w}^{\text {opt }}$ of parameter $\boldsymbol{w}$ through an optimization problem in $\boldsymbol{w}$ that requires to solve the stochastic boundary value problem and deduce an optimal prior model $\mathbb{V}^{\text {prior }}=\mathbb{V}^{\text {prior }}\left(\boldsymbol{w}^{\text {opt }}\right)$.

Step 3. Generation of $\nu_{\mathrm{KL}}$ independent realizations $\mathbb{V}^{\text {prior }}\left(\boldsymbol{w}^{\text {opt }}, \theta_{\ell}\right)$ for $\ell=$ $1, \ldots, \nu_{\mathrm{KL}}$ from the optimal prior model. Estimation of the covariance matrix $\left[C_{\text {Vprior }}\right]$ of the optimal prior model $\mathbb{V}^{\text {prior }}$. Solving $\left[C_{\text {Vprior }}\right] \mathbb{W}^{j}=\lambda_{j} \mathbb{W}^{j}$. From the statistical reduced-order optimal prior model $\mathbb{V}^{\text {prior }} \simeq \underline{\mathbb{V}}+\sum_{j=1}^{n} \sqrt{\lambda_{j}} \eta_{j}^{\text {prior }} \mathbb{W}^{j}$, deducing $\nu_{\mathrm{KL}}$ independent realizations $\boldsymbol{\eta}^{\text {prior }}\left(\theta_{\ell}\right)$ for $\ell=1, \ldots, \nu_{\mathrm{KL}}$ of random vector $\boldsymbol{\eta}^{\text {prior }}=\left(\eta_{1}^{\text {prior }}, \ldots, \eta_{n}^{\text {prior }}\right)$. It should be noted that the experimental realizations of $\boldsymbol{\eta}^{\text {prior }}$ associated with the partial experimental data cannot be constructed within the context introduced.

Step 4. Construction of the polynomial chaos expansion with deterministic vector-valued coefficients of the reduced-order optimal prior model $\boldsymbol{\eta}^{\text {prior }} \simeq$ $\boldsymbol{\eta}^{\text {chaos }}(N)$ with $\boldsymbol{\eta}^{\text {chaos }}(N)=\sum_{\alpha=1}^{N} \boldsymbol{y}^{\alpha} \Psi_{\alpha}(\boldsymbol{\Xi})$ and analyzing the convergence with respect to $N$. The optimal values $\left(\boldsymbol{y}^{1, \mathrm{opt}}, \ldots, \boldsymbol{y}^{N, \text { opt }}\right)$ of $\left(\boldsymbol{y}^{1}, \ldots, \boldsymbol{y}^{N}\right)$ are obtained using the maximum likelihood method with the independent realizations $\boldsymbol{\eta}^{\text {prior }}\left(\theta_{1}\right), \ldots, \boldsymbol{\eta}^{\text {prior }}\left(\theta_{\nu_{\mathrm{KL}}}\right)$. It should be noted that the corresponding optimization problem in high dimension can be solved thanks to the use of an adapted random search algorithm which will be detailed in Section 3.

Step 5. In general, the family of prior stochastic models which is introduced is not capable to perfectly represent the data (for instance, this can be the case for the mesoscale stochastic modeling of complex anisotropic and heterogeneous microstructures). It should be noted that the family of prior stochastic models does not take into account modeling errors but is introduced to model the random 
medium. Consequently, a posterior stochastic model must be introduced in order to improve the optimal prior stochastic model and in order to take into account the modeling errors induced by the choice of a prior stochastic model. Following the method of reduced polynomial chaos expansions with random coefficients of vector-valued random variables presented in [56] and in order to take into account model uncertainties, the optimal values $\left(\boldsymbol{y}^{1, \mathrm{opt}}, \ldots, \boldsymbol{y}^{N, \mathrm{opt}}\right)$ constructed in Step 4 are replaced by a family of random vectors $\left\{\boldsymbol{Y}^{1}, \ldots, \boldsymbol{Y}^{N}\right\}$ which are independent of the family of random variables $\left\{\Psi_{1}(\boldsymbol{\Xi}), \ldots, \Psi_{N}(\boldsymbol{\Xi})\right\}$. We then defined a posterior model $\mathbb{V}^{\text {post }}$ of $\mathbb{V}$ such that

$$
\mathbb{V}^{\text {post }}=\underline{\mathbb{V}}+\sum_{j=1}^{n} \sqrt{\lambda_{j}} \eta_{j}^{\text {post }} \mathbb{W}^{j}
$$

in which the posterior model $\boldsymbol{\eta}^{\text {post }}=\left(\eta_{1}^{\text {post }}, \ldots, \eta_{n}^{\text {post }}\right)$ is written as the following polynomial chaos expansion with random coefficients deduced from Eq. (4),

$$
\boldsymbol{\eta}^{\text {post }}=\sum_{\alpha=1}^{N} \boldsymbol{Y}^{\alpha} \Psi_{\alpha}(\boldsymbol{\Xi})
$$

The first equation in Eq. (3) clearly holds for the posterior model and the mean values of the random coefficients have to be such that

$$
E\left\{\boldsymbol{Y}^{\alpha}\right\}=\underline{\boldsymbol{y}}^{\alpha} \quad, \quad \text { for } \alpha=1, \ldots, N \quad,
$$

in which $\underline{\boldsymbol{y}}^{1}, \ldots, \underline{\boldsymbol{y}}^{N}$ are $N$ given vectors in $\mathbb{R}^{n}$. For the posterior model, the second equation in Eq. (3) does not hold. For all $\alpha$, the statistical fluctuations of $\boldsymbol{Y}^{\alpha}$ around the mean value $\underline{\boldsymbol{y}}^{\alpha}$ is controlled by a dimensionless positive number denoted by $\sigma_{\alpha}$. We then introduced the following simple model for the covariance matrix $\left[C_{\boldsymbol{Y}^{\alpha}}\right]$ of random vector $\boldsymbol{Y}^{\alpha}$,

$$
\left[C_{\boldsymbol{Y}^{\alpha}}\right]=\sigma_{\alpha}^{2} \underline{\boldsymbol{y}}^{\alpha} \underline{\boldsymbol{y}}^{\alpha T} \quad, \quad \text { for } \alpha=1, \ldots, N \quad .
$$

The probability distribution of the random vectors $\left\{\boldsymbol{Y}^{1}, \ldots, \boldsymbol{Y}^{N}\right\}$ is constructed using the maximum entropy principle $[49,25,26]$ under the constraints defined by the available information given by Eqs. (10) and (11). Consequently this probability distribution depends on unknown parameters $\underline{\boldsymbol{y}}^{1}, \ldots, \underline{\boldsymbol{y}}^{N}$ and $\sigma_{1}, \ldots, \sigma_{N}$ which can be identified using the maximum likelihood method with the experimental data $\boldsymbol{u}^{\exp , \ell}$ for $\ell=1, \ldots, \nu_{\text {exp }}$ for the posterior model $\boldsymbol{U}^{\text {post }}=\boldsymbol{h}\left(\mathbb{V}^{\text {post }}\right)$ of 
the observation vector (thus requiring to solve the stochastic boundary value problem). The corresponding optimization problem is then solved with an adapted algorithm for which the optimal solution is searched in the neighborhood of the optimal deterministic coefficients $\left(\boldsymbol{y}^{1, \mathrm{opt}}, \ldots, \boldsymbol{y}^{N, \mathrm{opt}}\right)$ identified in Step 4 and yields the optimal value $\left(\underline{\boldsymbol{y}}^{1, \mathrm{opt}}, \ldots, \underline{\boldsymbol{y}}^{N, \mathrm{opt}}\right)$ of $\left(\underline{\boldsymbol{y}}^{1}, \ldots, \underline{\boldsymbol{y}}^{N}\right)$ and the optimal value $\sigma_{1}^{\mathrm{opt}}$, $\ldots, \sigma_{N}^{\text {opt }}$ of $\sigma_{1}, \ldots, \sigma_{N}$.

\subsection{Developing the methodology}

Step 1. Introduction of a family of prior probability models of random vector $\mathbb{V}$. As we have explained in Section 1, the available experimental data are assumed to be not sufficient to perform a direct statistical estimation of the covariance matrix $\left[C_{\mathbb{V}}\right]$ that is necessary to construct the decomposition defined by Eq. (2). In addition, as we have also explained, this decomposition must have the capability to represent the required mathematical properties of $\left\{\left[\boldsymbol{A}\left(\boldsymbol{x}^{1}\right)\right], \ldots,\left[\boldsymbol{A}\left(\boldsymbol{x}^{N_{p}}\right)\right]\right\}$. To circumvent these two major difficulties, we propose to introduce a family of prior probabilistic models $\left\{\left[\boldsymbol{A}^{\text {prior }}(\boldsymbol{x} ; \boldsymbol{w})\right], \boldsymbol{x} \in \Omega\right\}$ of the matrix-valued random field $\{[\boldsymbol{A}(\boldsymbol{x})], \boldsymbol{x} \in \Omega\}$. We can then deduce a family of prior probability models $\mathbb{V}^{\text {prior }}(\boldsymbol{w})$ of random vector $\mathbb{V}$. This family of prior probability models is defined on a probability space $(\Theta, \mathcal{T}, \mathcal{P})$ and depends on the vector-valued parameter $\boldsymbol{w}$ belonging to an admissible set $\mathcal{C}_{\text {ad }}$. The knowledge of such a family of prior probability models means that the family of probability distributions $\left\{P_{\mathbb{V}}^{\text {prior }}(d v ; \boldsymbol{w}), \boldsymbol{w} \in \mathcal{C}_{\text {ad }}\right\}$ on $\mathbb{R}^{m_{\mathbb{V}}}$ of the family of random vectors $\left\{\mathbb{V}^{\text {prior }}(\boldsymbol{w}), \boldsymbol{w} \in\right.$ $\left.\mathcal{C}_{\text {ad }}\right\}$ is known. In addition, it is assumed that a generator of independent realizations $\mathbb{V}^{\text {prior }}\left(\theta_{1} ; \boldsymbol{w}\right), \ldots, \mathbb{V}^{\text {prior }}\left(\theta_{\nu_{\mathrm{KL}}} ; \boldsymbol{w}\right)$ for $\theta_{1}, \ldots, \theta_{\nu_{\mathrm{KL}}}$ belonging to $\Theta$ is available.

Step 2. Identification with the experimental data of an optimal prior model in the constructed family. Using the computational model (see Eq. (2)) and the family of prior probability models $\mathbb{V}^{\text {prior }}(\boldsymbol{w})$ of $\mathbb{V}$, we can construct the family $\left\{\boldsymbol{U}^{\text {prior }}(\boldsymbol{w}), \boldsymbol{w} \in \mathcal{C}_{\text {ad }}\right\}$ of random observation vectors such that

$$
\boldsymbol{U}^{\text {prior }}(\boldsymbol{w})=\boldsymbol{h}\left(\mathbb{V}^{\text {prior }}(\boldsymbol{w})\right) \quad, \quad \boldsymbol{w} \in \mathcal{C}_{\mathrm{ad}} .
$$

The optimal prior model is then obtained in finding the optimal value $\boldsymbol{w}^{\text {opt }}$ of $\boldsymbol{w}$ which minimizes an adapted "distance" between the family $\left\{\boldsymbol{U}^{\text {prior }}(\boldsymbol{w}), \boldsymbol{w} \in \mathcal{C}_{\text {ad }}\right\}$ of random observation vectors and the family of experimental data $\left\{\boldsymbol{u}^{\exp , 1}, \ldots\right.$,

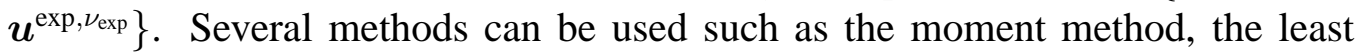
square method, the maximum likelihood method, etc (see [48, 57, 60]). It is assumed that the dimension of vector $\boldsymbol{w}$ is much less than $\nu_{\exp } \times m$. With such 
a hypothesis, a method such as the maximum likelihood is not really necessary and a method in the class of the least square method is generally sufficient and efficient. The optimal prior model is then constructed in solving the following optimization problem,

$$
\boldsymbol{w}^{\text {opt }}=\arg \min _{\boldsymbol{w} \in \mathcal{C}_{\mathrm{ad}}} J^{\text {prior }}(\boldsymbol{w}),
$$

in which the cost function $J^{\text {prior }}(\boldsymbol{w})$ can then be written, for instance, as

$$
J^{\text {prior }}(\boldsymbol{w})=\alpha\left\|\underline{\boldsymbol{U}}^{\text {prior }}(\boldsymbol{w})-\underline{\boldsymbol{U}}^{\exp }\right\|^{2}+(1-\alpha)\left\|\boldsymbol{\sigma}^{\text {prior }}(\boldsymbol{w})-\boldsymbol{\sigma}^{\exp }\right\|^{2},
$$

in which $\underline{\boldsymbol{U}}^{\text {prior }}(\boldsymbol{w})=\left(\underline{U}_{1}^{\text {prior }}(\boldsymbol{w}), \ldots, \underline{U}_{m}^{\text {prior }}(\boldsymbol{w})\right)$ and $\boldsymbol{\sigma}^{\text {prior }}(\boldsymbol{w})=\left(\sigma_{1}^{\text {prior }}(\boldsymbol{w}), \ldots\right.$, $\left.\sigma_{m}^{\text {prior }}(\boldsymbol{w})\right)$ are the mean value and the standard deviation of random vector $\boldsymbol{U}^{\text {prior }}(\boldsymbol{w})=$ $\left(U_{1}^{\text {prior }}(\boldsymbol{w}), \ldots, U_{m}^{\text {prior }}(\boldsymbol{w})\right)=\boldsymbol{h}\left(\mathbb{V}^{\text {prior }}(\boldsymbol{w})\right)$ with $\sigma_{k}^{\text {prior }}(\boldsymbol{w})$ the standard deviation of the real-valued random variable $U_{k}^{\text {prior }}(\boldsymbol{w})$ and where $\underline{\boldsymbol{U}}^{\exp }=\left(\underline{U}_{1}^{\exp }, \ldots, \underline{U}_{m}^{\exp }\right)$ and $\boldsymbol{\sigma}^{\exp }=\left(\sigma_{1}^{\exp }, \ldots, \sigma_{m}^{\exp }\right)$ are such that

$$
\underline{U}_{k}^{\exp }=\frac{1}{\nu_{\text {exp }}} \sum_{\ell=1}^{\nu_{\text {exp }}} u_{k}^{\exp , \ell} \quad, \quad \sigma_{k}^{\exp }=\left\{\frac{1}{\nu_{\text {exp }}} \sum_{\ell=1}^{\nu_{\text {exp }}}\left(u_{k}^{\exp , \ell}\right)^{2}-\left(\underline{U}_{k}^{\exp }\right)^{2}\right\}^{1 / 2} .
$$

In Eq. (14)), $\alpha$ is chosen in $[0,1]$ and can be taken, for instance, as $\alpha=0.5$.

Step 3. Construction of the statistical reduced-order optimal prior model. As it is explained in Section 1, a statistical reduced-order optimal prior model must be constructed using the KL expansion in order to be able to construct an efficient polynomial chaos expansion of the optimal prior model. This step then consists in constructing a reduced-order representation of the type defined by Eq. (2)) for the optimal prior model $\mathbb{V}^{\text {prior }}\left(\boldsymbol{w}^{\text {opt }}\right)$ that we will simply denote by $\mathbb{V}^{\text {prior }}$ The mean value of $\mathbb{V}^{\text {prior }}\left(\boldsymbol{w}^{\mathrm{opt}}\right)$ is $\underline{\mathbb{V}}=E\left\{\mathbb{V}^{\text {prior }}\left(\boldsymbol{w}^{\mathrm{opt}}\right)\right\}$ and its symmetric positive-definite $\left(m_{\mathbb{V}} \times m_{\mathbb{V}}\right)$ real covariance matrix is $\left[C_{\mathbb{V} p r i o r}\right]=E\left\{\left(\mathbb{V}^{\text {prior }}\left(\boldsymbol{w}^{\mathrm{opt}}\right)-\right.\right.$ $\left.\underline{\mathbb{V}})\left(\mathbb{V}^{\text {prior }}\left(\boldsymbol{w}^{\mathrm{opt}}\right)-\underline{\mathbb{V}}\right)^{T}\right\}$. These second-order moments are estimated using $\nu_{\mathrm{KL}}$ independent realizations $\mathbb{V}^{\text {prior }}\left(\boldsymbol{w}^{\mathrm{opt}}, \theta_{\ell}\right)$ for $\ell=1, \ldots \nu_{\mathrm{KL}}$ generated with the optimal prior probabilistic model. The dominant eigenspace of the eigenvalue problem $\left[C_{\mathbb{V}^{p r i o r}}\right] \mathbb{W}^{j}=\lambda_{j} \mathbb{W}^{j}$ is then constructed. Let $[\mathbb{W}]=\left[\mathbb{W}^{1} \ldots \mathbb{W}^{n}\right]$ be the $\left(m_{\mathbb{V}} \times n\right)$ real matrix of the $n$ eigenvectors associated with the $n$ largest eigenvalues $\lambda_{1} \geq \lambda_{2} \geq \ldots \geq \lambda_{n}>0$ such that $[\mathbb{W}]^{T}[\mathbb{W}]=\left[I_{n}\right]$. The statistical reduced-order optimal prior model is then written as

$$
\mathbb{V}^{\text {prior }} \simeq \underline{\mathbb{V}}+\sum_{j=1}^{n} \sqrt{\lambda_{j}} \eta_{j}^{\text {prior }} \mathbb{W}^{j}
$$


in which the optimal prior model $\boldsymbol{\eta}^{\text {prior }}=\left(\eta_{1}^{\text {prior }}, \ldots, \eta_{n}^{\text {prior }}\right)$ is a second-order centered random variable with values in $\mathbb{R}^{n}$ such that

$$
E\left\{\boldsymbol{\eta}^{\text {prior }}\right\}=\mathbf{0} \quad, \quad E\left\{\boldsymbol{\eta}^{\text {prior }}\left(\boldsymbol{\eta}^{\text {prior }}\right)^{T}\right\}=\left[I_{n}\right] .
$$

The mean-square convergence of the right-hand side in Eq. (16)) with respect to the reduced order $n$ is studied in constructing the error function

$$
n \mapsto \operatorname{err}(n)=1-\frac{\sum_{j=1}^{n} \lambda_{j}}{\operatorname{tr}\left[C_{\mathbb{V p r i o r}}\right]},
$$

which is a monotonic decreasing function from $\left\{1, \ldots, m_{\mathbb{V}}\right\}$ into $[0,1]$ and such that $\operatorname{err}\left(m_{\mathbb{V}}\right)=0$. The $\nu_{\mathrm{KL}}$ independent realizations $\boldsymbol{\eta}^{\text {prior }}\left(\theta_{1}\right), \ldots, \boldsymbol{\eta}^{\text {prior }}\left(\theta_{\nu_{\mathrm{KL}}}\right)$ are deduced from the realizations $\mathbb{V}^{\text {prior }}\left(\boldsymbol{w}^{\mathrm{opt}}, \theta_{1}\right), \ldots, \mathbb{V}^{\text {prior }}\left(\boldsymbol{w}^{\mathrm{opt}}, \theta_{\nu_{\mathrm{KL}}}\right)$ using, for $j=$ $1, \ldots, n$ and for $\ell=1, \ldots \nu_{\mathrm{KL}}$, the equation

$$
\eta_{j}^{\text {prior }}\left(\theta_{\ell}\right)=\frac{1}{\sqrt{\lambda_{j}}}\left(\mathbb{V}^{\text {prior }}\left(\boldsymbol{w}^{\text {opt }}, \theta_{\ell}\right)-\underline{\mathbb{V}}\right)^{T} \mathbb{W}^{j}
$$

Step 4. Construction of the polynomial chaos expansion with deterministic vector-valued coefficients of the reduced-order optimal prior model. This step consists in constructing an approximation of the reduced optimal prior model $\boldsymbol{\eta}^{\text {prior }}$ by a polynomial chaos expansion $\boldsymbol{\eta}^{\text {chaos }}(N)=\left(\eta_{1}^{\text {chaos }}(N), \ldots, \eta_{n}^{\text {chaos }}(N)\right)$ such that

$$
\boldsymbol{\eta}^{\text {prior }} \simeq \boldsymbol{\eta}^{\text {chaos }}(N) \quad, \quad \boldsymbol{\eta}^{\text {chaos }}(N)=\sum_{\alpha=1}^{N} \boldsymbol{y}^{\alpha} \Psi_{\alpha}(\boldsymbol{\Xi})
$$

for which $\nu_{\mathrm{KL}}$ independent realizations $\boldsymbol{\eta}^{\text {prior }}\left(\theta_{1}\right), \ldots, \boldsymbol{\eta}^{\text {prior }}\left(\theta_{\nu_{\mathrm{KL}}}\right)$ of $\boldsymbol{\eta}^{\text {prior }}$ are known from Eq. (19) of Step 3. In Eq. (20)), the symbol " " means that the mean-square convergence is reached for $N$ sufficiently large and the deterministic vector-valued coefficients which have to be identified are the $N$ vectors $\boldsymbol{y}^{1}, \ldots, \boldsymbol{y}^{N}$ in $\mathbb{R}^{n}$. The random variables $\Psi_{\alpha}(\boldsymbol{\Xi})$ (the polynomial chaos) are defined in Section 1-(4). Taking into account Eqs. (6) and (17), it can be deduced that vectors $\boldsymbol{y}^{1}, \ldots, \boldsymbol{y}^{N}$ must verify the following equation,

$$
\sum_{\alpha=1}^{N} \boldsymbol{y}^{\alpha} \boldsymbol{y}^{\alpha T}=\left[I_{n}\right]
$$


For all $j=1, \ldots, n$, let $e \mapsto p_{\eta_{j}^{\text {prior }}}(e)$ be the probability density function of the random variable $\eta_{j}^{\text {prior }}$ estimated with the optimal prior model constructed in Step 3. For all $\boldsymbol{y}^{1}, \ldots, \boldsymbol{y}^{N}$ fixed in $\mathbb{R}^{n}$ and satisfying Eq. (21)) and for all $j=$ $1, \ldots, n$, let $e \mapsto p_{\eta_{j}^{\text {chaos }}(N)}\left(e ; \boldsymbol{y}^{1}, \ldots, \boldsymbol{y}^{N}\right)$ be the probability density function of random variable $\eta_{j}^{\text {chaos }}(N)$ estimated with the polynomial chaos expansion defined by Eq. (20). The convergence of the sequence of random vectors $\left\{\boldsymbol{\eta}^{\text {chaos }}(N)\right\}_{N}$ towards $\boldsymbol{\eta}^{\text {prior }}$ can be controlled as follows. Since $N$ depends on $N_{g}$ and $N_{d}, N$ is also rewritten as $N=h\left(N_{g}, N_{d}\right)$. For each component $\eta_{j}^{\text {chaos }}(N)$, we introduce the $L^{1}$-log error function defined by

$$
\operatorname{err}_{j}\left(N_{g}, N_{d}\right)=\int_{\mathrm{BI}_{j}}\left|\log _{10} p_{\eta_{j}^{\text {prior }}}(e)-\log _{10} p_{\eta_{j}^{\text {chaos }}(N)}\left(e ; \boldsymbol{y}^{1}, \ldots, \boldsymbol{y}^{N}\right)\right| d e,
$$

in which $\mathrm{BI}_{j}$ is a bounded interval of the real line which is adapted to the problem. Let $\widehat{p}_{\eta_{j}^{\text {prior }}}(e)$ be the estimation of the probability density function $p_{\eta_{j}^{\text {prior }}}(e)$ carried out using the kernel density estimation method [4] with the independent realizations $\eta_{j}^{\text {prior }}\left(\theta_{1}\right), \ldots, \eta_{j}^{\text {prior }}\left(\theta_{\nu_{\mathrm{KL}}}\right)$. Let $\left[\widehat{a}_{j}^{\text {prior }}, \widehat{b}_{j}^{\text {prior }}\right]$ be the support of $e \mapsto \widehat{p}_{\eta_{j}^{\text {prior }}}(e)$. Similarly, for a given value of $\boldsymbol{y}^{1}, \ldots, \boldsymbol{y}^{N}$, let $\widehat{p}_{\eta_{j}^{\text {chaos }}(N)}\left(e ; \boldsymbol{y}^{1}, \ldots, \boldsymbol{y}^{N}\right)$ be the estimation of the probability density function $p_{\eta_{j}^{\text {chaos }}(N)}\left(e ; \boldsymbol{y}^{1}, \ldots, \boldsymbol{y}^{N}\right)$ carried out using $\nu_{\text {chas }}$ independent realizations of the normalized Gaussian vector $\boldsymbol{\Xi}$ and Eq. (20). Let $\left[\widehat{a}_{j}^{\text {chaos }}(N), \widehat{b}_{j}^{\text {chaos }}(N)\right]$ be the support of $e \mapsto \widehat{p}_{\eta_{j}^{\text {chas }}(N)}\left(e ; \boldsymbol{y}^{1}, \ldots, \boldsymbol{y}^{N}\right)$. The bounded interval $\mathrm{BI}_{j}=\left[\widehat{a}_{j}, \widehat{b}_{j}\right]$ is defined by $\widehat{a}_{j}=\max \left\{\widehat{a}_{j}^{\text {prior }}, \widehat{a}_{j}^{\text {chaos }}(N)\right\}$ and $\widehat{b}_{j}=\min \left\{\widehat{b}_{j}^{\text {prior }}, \widehat{b}_{j}^{\text {chas }}(N)\right\}$. For the random vector $\boldsymbol{\eta}^{\text {chaos }}(N)$, the $L^{1}-\log$ error function is denoted as $\operatorname{err}\left(N_{g}, N_{d}\right)$ and is defined by

$$
\operatorname{err}\left(N_{g}, N_{d}\right)=\frac{1}{n} \sum_{j=1}^{n} \operatorname{err}_{j}\left(N_{g}, N_{d}\right)
$$

The unusual $L^{1}-\log$ error function defined by Eq. (22) has been introduced in order to measure the errors of the very small values of the probability density function (the tails of the probability density function).

A natural way to solve such a problem is to use the maximum likelihood method $[48,57,60]$ as done in $[9,10,7,1]$. Taking into account that the dependent random variables $\eta_{1}^{\text {prior }}, \ldots, \eta_{n}^{\text {prior }}$ are not correlated, the following approximation $\mathcal{L}\left(\boldsymbol{y}^{1}, \ldots, \boldsymbol{y}^{N}\right)$ of the log-likelihood function is introduced

$$
\mathcal{L}\left(\boldsymbol{y}^{1}, \ldots, \boldsymbol{y}^{N}\right)=\sum_{j=1}^{n} \sum_{\ell=1}^{\nu_{\mathrm{KL}}} \log _{10} p_{\eta_{j}^{\text {chaos }}(N)}\left(\eta_{j}^{\text {prior }}\left(\theta_{\ell}\right) ; \boldsymbol{y}^{1}, \ldots, \boldsymbol{y}^{N}\right)
$$


The optimal value $\left(\boldsymbol{y}^{1, \mathrm{opt}}, \ldots, \boldsymbol{y}^{N, \mathrm{opt}}\right)$ of $\left(\boldsymbol{y}^{1}, \ldots, \boldsymbol{y}^{N}\right)$ is then given by

$$
\left(\boldsymbol{y}^{1, \mathrm{opt}}, \ldots, \boldsymbol{y}^{N, \mathrm{opt}}\right)=\arg \max _{\left(\boldsymbol{y}^{1}, \ldots, \boldsymbol{y}^{N}\right) \in \mathcal{C}_{\mathrm{ad}}^{N}} \mathcal{L}\left(\boldsymbol{y}^{1}, \ldots, \boldsymbol{y}^{N}\right)
$$

in which $\mathcal{C}_{\text {ad }}^{N}$ is such that

$$
\mathcal{C}_{\mathrm{ad}}^{N}=\left\{\left(\boldsymbol{y}^{1}, \ldots, \boldsymbol{y}^{N}\right) \in\left(\mathbb{R}^{n}\right)^{N}, \sum_{\alpha=1}^{N} \boldsymbol{y}^{\alpha} \boldsymbol{y}^{\alpha T}=\left[I_{n}\right]\right\}
$$

For the high-dimension case, that is to say for $n$ and $N$ very large, solving the optimization problem defined by Eqs. (25) and (26) is a challenging problem which has been solved in the last decade only for small values of $n$ and $N$. There are two major difficulties.

(a) The first one is related to the construction of the log-likelihood function which requires to generate $\nu_{\text {chaos }}$ independent realizations $\Psi_{\alpha}\left(\boldsymbol{\Xi}\left(\theta_{\ell}^{\prime}\right)\right)$ for $\alpha=$ $1, \ldots, N$ and $\ell=1, \ldots, \nu_{\text {chaos }}$ of $\Psi_{\alpha}(\boldsymbol{\Xi})$ for $\alpha=1, \ldots, N$ with high degree $N_{d}$ of the polynomials $\Psi_{\alpha}$. We then introduce the $\left(\nu_{\text {chaos }} \times N\right)$ real matrix $[\Psi]$ such that $[\Psi]_{\ell \alpha}=\Psi_{\alpha}\left(\boldsymbol{\Xi}\left(\theta_{\ell}^{\prime}\right)\right)$. When the recurrence algebraic formula is used to compute matrix $[\Psi]$, the numerical noise increases with the degree $N_{d}$ of the polynomials and the fundamental orthogonality condition defined by Eq. (6) is lost. To circumvent this major difficulty, we use a novel method (see [54]) to compute matrix $[\Psi]$ which allows the orthogonality conditions defined by Eq. (6) to be kept for any values of $N_{g}$ and $N_{d}$. This novel method consists (1) in constructing the realizations of the multivariate monomials using a generator of independent realizations of the germs whose probability distribution is the given arbitrary measure and (2) in performing an orthogonalization of the realizations of the multivariate monomials with an algorithm different from the Gram-Schmidt orthogonalization algorithm which is not stable in high dimension.

(b) The second major difficulty is related to the choice of the optimization algorithm which allows the problem defined by Eq. (25) to be solved with a reasonable CPU time for high dimension, the constraint $\sum_{\alpha=1}^{N} \boldsymbol{y}^{\alpha} \boldsymbol{y}^{\alpha T}=\left[I_{n}\right]$ having to be exactly verified. We then propose a new random search algorithm adapted to this problem which is very efficient and which is detailed in Section 3.

Step 5. Construction of the probability distribution of random coefficients of the polynomial chaos expansion and identification of the parameters using experimental data. The probability density function $p_{\boldsymbol{Y}^{1} \ldots \boldsymbol{Y}^{N}}\left(\boldsymbol{y}^{1}, \ldots, \boldsymbol{y}^{N}\right)$ with respect 
to the Lebesgue measure $d \boldsymbol{y}^{1} \ldots d \boldsymbol{y}^{N}$ on $\mathbb{R}^{n} \times \ldots \times \mathbb{R}^{n}$ ( $N$ times) of the $\mathbb{R}^{n}$ valued random variables $\boldsymbol{Y}^{1}, \ldots, \boldsymbol{Y}^{N}$ is constructed using the maximum entropy principle under the constraints defined by Eqs. (10) and (11). For $\alpha=1, \ldots, N$, let $\boldsymbol{\mu}^{\alpha}$ in $\mathbb{R}^{n}$ and $\left[\Lambda^{\alpha}\right]$ in the set of all the positive-definite symmetric $(n \times n)$ real matrices be the Lagrange multipliers associated with the constraints defined by Eq. (10) and (11) respectively. Then the probability density function which maximizes the entropy is written as

$$
p_{\boldsymbol{Y}^{1} \ldots \boldsymbol{Y}^{N}}\left(\boldsymbol{y}^{1}, \ldots, \boldsymbol{y}^{N}\right)=c_{0} \Pi_{\alpha=1}^{N} \exp \left\{-<\boldsymbol{\mu}^{\alpha}, \boldsymbol{y}^{\alpha}>-<\left[\Lambda^{\alpha}\right] \boldsymbol{y}^{\alpha}, \boldsymbol{y}^{\alpha}>\right\},
$$

in which $c_{0}$ is the constant of normalization and where the brackets denote the Euclidean inner product in $\mathbb{R}^{n}$. From Eq. (27), it can be deduced that $\boldsymbol{Y}^{1}, \ldots, \boldsymbol{Y}^{N}$ are independent Gaussian random variables with values in $\mathbb{R}^{n}$. For all $\alpha=$ $1, \ldots, N$, the mean value of random vector $\boldsymbol{Y}^{\alpha}$ is $E\left\{\boldsymbol{Y}^{\alpha}\right\}=\underline{\boldsymbol{y}}^{\alpha}$ (see Eq. (10)) and its covariance matrix $\left[C_{\boldsymbol{Y}^{\alpha}}\right]=E\left\{\left(\boldsymbol{Y}^{\alpha}-\underline{\boldsymbol{y}}^{\alpha}\right)\left(\boldsymbol{Y}^{\alpha}-\underline{\boldsymbol{y}}^{\alpha}\right)^{T}\right\}$ is given by Eq. (11). It can easily be verified that for all $\alpha$, the Gaussian random vector $\boldsymbol{Y}^{\alpha}=\left(Y_{1}^{\alpha}, \ldots, Y_{n}^{\alpha}\right)$ can be written as

$$
Y_{j}^{\alpha}=\underline{y}_{j}^{\alpha}\left(1+\sigma_{\alpha} \mathcal{N}_{j}^{\alpha}\right)
$$

in which $\left\{\mathcal{N}_{j}^{\alpha}, \alpha=1, \ldots, N ; j=1, \ldots, n\right\}$ is a family of independent centered Gaussian random variables with unit standard deviation. From Eqs. (9), (6) and (28), it can be deduced that

$$
\left[E\left\{\boldsymbol{\eta}^{\text {post }}\left(\boldsymbol{\eta}^{\text {post }}\right)^{T}\right\}\right]_{j k}=\sum_{\alpha=1}^{N}\left(1+\sigma_{\alpha}^{2} \delta_{j k}\right) \underline{y}_{j}^{\alpha} \underline{y}_{k}^{\alpha} .
$$

The family $\left\{\underline{\boldsymbol{y}}^{1}, \ldots, \underline{\boldsymbol{y}}^{N}\right\}$ of vectors and the family $\left\{\sigma_{1}, \ldots, \sigma_{N}\right\}$ of real numbers could be chosen such that $\left.E\left\{\boldsymbol{\eta}^{\text {post }}\left(\boldsymbol{\eta}^{\text {post }}\right)^{T}\right\}\right]=\left[I_{n}\right]$ but, with such a choice, the posterior model would be very close to the prior model without the possibility to update the prior model to get a better representation of the experimental observations. Consequently, this condition will not be kept and for the posterior model we will have $\left.E\left\{\boldsymbol{\eta}^{\text {post }}\left(\boldsymbol{\eta}^{\text {post }}\right)^{T}\right\}\right] \neq\left[I_{n}\right]$. In order to simplify notation, we introduce the vector $\mathcal{Y}$ belonging to $\left(\mathbb{R}^{n}\right)^{N}=\mathbb{R}^{n} \times \ldots \times \mathbb{R}^{n}$ ( $N$ times) such that $\underline{\mathcal{Y}}=\left(\underline{\boldsymbol{y}}^{1}, \ldots, \underline{\boldsymbol{y}}^{N}\right)$ and the vector $\boldsymbol{\sigma}$ belonging to $\mathbb{R}^{N}$ such that $\boldsymbol{\sigma}=\left(\sigma_{1}, \ldots, \sigma_{N}\right)$. Comparing this probabilistic model with the general method of reduced chaos decompositions with random coefficients of vector-valued random variables presented in [56], it can be seen that this probabilistic model corresponds to the chaos 
expansion of the random coefficients limited to the first-order expansion (Gaussian approximation).

As previously explained, an optimal value $\underline{\mathcal{Y}}^{\text {opt }}=\left(\underline{\boldsymbol{y}}^{1, \mathrm{opt}}, \ldots, \underline{\boldsymbol{y}}^{N, \mathrm{opt}}\right)$ of $\underline{\mathcal{Y}}=$ $\left(\underline{\boldsymbol{y}}^{1}, \ldots, \underline{\boldsymbol{y}}^{N}\right)$ and the optimal value $\boldsymbol{\sigma}^{\mathrm{opt}}=\left(\sigma_{1}^{\mathrm{opt}}, \ldots, \sigma_{N}^{\mathrm{opt}}\right)$ of $\boldsymbol{\sigma}=\left(\sigma_{1}, \ldots, \sigma_{N}\right)$ are calculated in order to minimize an adapted "distance" between the experimental data $\left\{\boldsymbol{u}^{\exp , 1}, \ldots, \boldsymbol{u}^{\exp , \nu_{\exp }}\right\}$ and the posterior model $\boldsymbol{U}^{\text {post }}=\boldsymbol{h}\left(\mathbb{V}^{\text {post }}\right)$ of the observation vector. Since low-probability levels must be correctly identified, we propose to use a cost function based on an $L^{1}-\log$ error function of the probability density functions and then, the optimal posterior model is constructed in solving the following optimization problem,

$$
\begin{gathered}
\left(\underline{\mathcal{Y}}^{\text {opt }}, \boldsymbol{\sigma}^{\text {opt }}\right)=\arg \min _{(\underline{\mathcal{Y}}, \boldsymbol{\sigma})} \mathcal{J}^{\text {post }}(\underline{\mathcal{Y}}, \boldsymbol{\sigma}) \\
\mathcal{J}^{\text {post }}(\underline{\mathcal{Y}}, \boldsymbol{\sigma})=\frac{1}{m} \sum_{k=1}^{m} \int_{\mathrm{BI}_{k}}\left|\log _{10} p_{U_{k}^{\text {post }}}(u ; \underline{\mathcal{Y}}, \boldsymbol{\sigma})-\log _{10} \widehat{p}_{U_{k}^{\exp }}\left(u ; \nu_{\exp }\right)\right| d u
\end{gathered}
$$

In Eq. (31)), $\widehat{p}_{U_{k}}^{\exp }\left(u ; \nu_{\exp }\right)$ denotes an estimation of the probability density function of $U_{k}^{\text {exp }}$ carried out using the kernel density estimation method [4] with the experimental data $u_{k}^{\exp , \ell}$ for $\ell=1, \ldots, \nu_{\text {exp }}$. Let $\left[\widehat{a}_{k}, \widehat{b}_{k}\right]$ be the support of $u \mapsto$ $\widehat{p}_{U_{k}}^{\exp }\left(u ; \nu_{\exp }\right)$. Then the bounded interval $\mathrm{BI}_{k}=\left[a_{k}, b_{k}\right]$ of the real line is chosen such that $a_{k}=\max \left\{\min _{\ell}\left(u_{k}^{\exp , \ell}\right), \widehat{a}_{k}\right\}$ and $b_{k}=\min \left\{\max _{\ell}\left(u_{k}^{\exp , \ell}\right), \widehat{b}_{k}\right\}$. It should be noted that the estimation $\widehat{p}_{U_{k}^{\exp }}\left(u ; \nu_{\exp }\right)$ of $p_{U_{k}^{\exp }}(u)$ is generally not converged because $\nu_{\text {exp }}$ is not sufficiently large. Nevertheless, this estimator "summarizes" the experimental data and is an experimental quantity that we propose to use for constructing the cost function.

The probability density function $u \mapsto p_{U_{k}^{\text {post }}}(u ; \underline{\mathcal{Y}}, \boldsymbol{\sigma})$ of the real-valued random variable $U_{k}^{\text {post }}(\underline{\mathcal{Y}}, \boldsymbol{\sigma})=\left\{\boldsymbol{h}\left(\mathbb{V}^{\text {post }}(\underline{\mathcal{Y}}, \boldsymbol{\sigma})\right)\right\}_{k}$ of the posterior model is calculated solving the stochastic boundary value problem for which the random vector $\left.\mathbb{V}^{\text {post }}(\underline{\mathcal{Y}}, \boldsymbol{\sigma})\right)$ is defined by Eqs. (8) and (9) and using the kernel density estimation method. The optimization problem defined by Eq. (30) is then solved with an adapted algorithm for which the optimal solution is searched in the neighborhood of the optimal deterministic coefficients $\left(\boldsymbol{y}^{1, \mathrm{opt}}, \ldots, \boldsymbol{y}^{N, \text { opt }}\right)$ identified in Step 4 and yields the optimal value $\underline{\mathcal{Y}}^{\text {opt }}=\left(\underline{\boldsymbol{y}}^{1, \mathrm{opt}}, \ldots, \underline{\boldsymbol{y}}^{N, \mathrm{opt}}\right)$ of $\underline{\mathcal{Y}}=\left(\underline{\boldsymbol{y}}^{1}, \ldots, \underline{\boldsymbol{y}}^{N}\right)$. The algorithm adapted to this optimization problem is detailed in Section 3. 


\subsection{Remarks concerning the proposed methodology}

\subsubsection{Remark concerning the prior and the posterior stochastic models}

For a given set of available experimental data, the best construction which can be made is the construction (1) which takes into account all the available theoretical information related to the tensor-valued random field and (2) which reproduces the set of the available experimental data in a statistical sense. The family of prior models which depends on a vector-valued parameter with a very low dimension must span the larger possible subset of all the admissible tensor-valued random fields. The construction of such a family must then take into account all the available theoretical information such as the ellipticity condition. Nevertheless, since the optimal prior model is constructed in identifying a family which has only a few free parameters, this optimal prior model belongs to a subset which is generally not enough big to perfectly represent the available experimental data. Consequently, it is necessary to construct a posterior model to better represent the experimental data, that is to say, it is necessary to construct a representation of the optimal prior model which is capable to span a larger subset containing the available data. The proposed solution consists in constructing the polynomial chaos representation of the optimal prior model. Such a polynomial chaos expansion is capable to represent any tensor-valued random field in modifying its coefficients and then has the capability to fit all the experimental data in a statistical sense. Nevertheless, a direct construction of such polynomial chaos expansion would not be realistic. This is the reason why, in Step 4, the polynomial chaos expansion of the optimal prior model is constructed and then, in Step 5, the posterior model is constructed in replacing the deterministic coefficients of the polynomial chaos expansion by random coefficients. Clearly, better will be the optimal prior model, less will be the numerical cost required to fit the probability distributions of the random coefficients in Step 5.

\subsubsection{Remark concerning the choice of the length $N_{g}$ of the germ}

Let us consider an uncertain computational model for which the stochastic modeling of uncertain parameters is performed by introducing $N_{g}$ independent random variables (after having applied a KL statistical reduction and a nonlinear transformation of independent random variables for which the probability measures are known). Clearly, for the direct problem consisting in analyzing the propagation of uncertainties through the computational model, the length of the germ of the polynomial chaos expansion of the random response of this stochastic computational model, must be chosen as $N_{g}$. 
In this paper, the stochastic inverse problem is considered. The uncertain parameter is a random field for which the probabilistic model is unknown and must be identified. Using the KL statistical reduction and then the polynomial chaos expansion of the $\mathbb{R}^{n}$-valued random variable $\eta^{\text {prior }}$, there are three unknown parameters $n, N_{g}$ and $N_{d}$, which must be selected to get a good convergence of the representation. The value of parameter $n$ is defined in studying the error function $n \mapsto \operatorname{err}(n)$ defined by Eq. (18) which is based on a mean-square convergence. The value of $n$ is then independent from the value of $N_{g}$ and $N_{d}$. Now, the value of $N_{g}$ and $N_{d}$ must be defined in order that the convergence of the polynomial chaos expansion of the $\mathbb{R}^{n}$-valued random variable $\boldsymbol{\eta}^{\text {prior }}$ be reached. Such a convergence is studied using the unusual $L^{1}-\log$ error function $\left(N_{g}, N_{d}\right) \mapsto \operatorname{err}\left(N_{g}, N_{d}\right)$ defined by Eqs. (22) and (23). It should be noted that this error function is better than the mean-square error function and allows the convergence of the probability function to be controlled over all the range of the large values and the very small values of the probability levels (this means that this error function allows the tail of the probability density functions to be correctly fitted). Consequently, the maximum degree $N_{d}$ of the polynomial chaos must be sufficiently high to get the convergence of the representation when the random field is any non-Gaussian random field (a random field which is unknown and which must be identified without any information about the tails of the system of marginal probability distributions which define the probability law of the non-Gaussian random field). In addition, the introduction of a very large number $N$ of polynomial chaos $\left\{\Psi_{\alpha}(\boldsymbol{\Xi}), \alpha=1, \ldots, N\right\}$ induced by the use of a high value of $N_{d}$ coupled with the use of a significant value of $N_{g}$, is equivalent to the introduction of a very large number of uncorrelated random variables due to the orthogonal property defined by Eq. (6).

For the stochastic inverse problem under consideration, this analysis shows that, there is no reason to set a priori a value for $N_{g}$ or a value for $N_{d}$ which are strongly dependent. The optimal values of $N_{g}$ and $N_{d}$ must be determined using the $L^{1}-\log$ error function and there is no reason to set the value of $N_{g}$ to the value $n$.

\section{Algorithms}

3.1. Algorithm for the identification of the deterministic coefficients of the polynomial chaos expansion (Step4)

In a first stage, we show that the initial optimization problem defined by Eqs. (25) and (26) can be reformulated as a set of recurrent optimization prob- 
lems relative to each coordinate and depending only on the chaos coefficients for each coordinate. In a second stage, we give some details concerning the random search algorithm applied to the reformulated optimization problem.

\subsubsection{Reformulation of the initial optimization problem as a set of recurrent op- timization problems for each coordinate}

In this subsection, we present a reformulation of the optimization problem defined by Eqs. (25) for which the admissible set $\mathcal{C}_{\text {ad }}^{N}$ is defined by Eq. (26). Let $\boldsymbol{z}^{1}, \ldots, \boldsymbol{z}^{n}$ be $n$ vectors in $\mathbb{R}^{N}$ such that $\boldsymbol{z}^{j}=\left(z_{1}^{j}, \ldots, z_{N}^{j}\right) \in \mathbb{R}^{N}$ with $z_{\alpha}^{j}=y_{j}^{\alpha}$ for all $j=1, \ldots, n$ and for all $\alpha=1, \ldots, N$. It is assumed that $N \geq n$. From Eq. (21), it can be deduced that

$$
<\boldsymbol{z}^{j}, \boldsymbol{z}^{k}>=\delta_{j k} \quad \text { for all } j \text { and } k \text { in }\{1, \ldots n\} \quad \text {. }
$$

The optimization problem defined by Eqs. (25) and (26) can then be rewritten as follows. The optimal value $\left(\boldsymbol{y}^{1, \mathrm{opt}}, \ldots, \boldsymbol{y}^{N, \mathrm{opt}}\right)$ is such that $y_{j}^{\alpha, \text { opt }}=z_{\alpha}^{j, \mathrm{opt}}$ in which $\left(\boldsymbol{z}^{1, \mathrm{opt}}, \ldots, \boldsymbol{z}^{n, \mathrm{opt}}\right)$ is given by

$$
\left(\boldsymbol{z}^{1, \mathrm{opt}}, \ldots, \boldsymbol{z}^{n, \mathrm{opt}}\right)=\arg \max _{\left(\boldsymbol{z}^{1}, \ldots, \boldsymbol{z}^{n}\right) \in \mathcal{C}_{\mathrm{ad}}^{n}} \mathcal{L}\left(\boldsymbol{z}^{1}, \ldots, \boldsymbol{z}^{n}\right)
$$

in which $\mathcal{C}_{\text {ad }}^{n}$ is such that

$$
\mathcal{C}_{\mathrm{ad}}^{n}=\left\{\left(\boldsymbol{z}^{1}, \ldots, \boldsymbol{z}^{n}\right) \in\left(\mathbb{R}^{N}\right)^{n},<\boldsymbol{z}^{j}, \boldsymbol{z}^{k}>=\delta_{j k}\right\},
$$

and where $\mathcal{L}\left(\boldsymbol{z}^{1}, \ldots, \boldsymbol{z}^{n}\right)$ is defined by

$$
\mathcal{L}\left(\boldsymbol{z}^{1}, \ldots, \boldsymbol{z}^{n}\right)=\sum_{j=1}^{n} \sum_{\ell=1}^{\nu_{\mathrm{KL}}} \log _{10} p_{\eta_{j}^{\text {chaos }}(N)}\left(\eta_{j}^{\text {prior }}\left(\theta_{\ell}\right) ; \boldsymbol{z}^{j}\right)
$$

It should be noted that the probability density function $p_{\eta_{j}^{\text {chaos }}(N)}\left(\eta_{j}^{\text {prior }}\left(\theta_{\ell}\right) ; \boldsymbol{z}^{j}\right)$ depends only on $\boldsymbol{z}^{j}$ because equation $\boldsymbol{\eta}^{\text {chaos }}(N)=\sum_{\alpha=1}^{N} \boldsymbol{y}^{\alpha} \Psi_{\alpha}(\boldsymbol{\Xi})$ can be rewritten as $\eta_{j}^{\text {chaos }}(N)=\sum_{\alpha=1}^{N} z_{\alpha}^{j} \Psi_{\alpha}(\boldsymbol{\Xi})$ and can then directly be estimated. Let $\mathcal{J}$ be the set of all the permutations of the first $n$ positive integers $\{1, \ldots, n\}$. A generic point in $\mathcal{J}$ is then denoted by $\left\{j_{1}, \ldots, j_{n}\right\}$. For a fixed point $\left\{j_{1}, \ldots, j_{n}\right\}$ in $\mathcal{J}$, the following recurrent optimization problems $\mathcal{P}_{j_{1}}, \ldots, \mathcal{P}_{j_{n}}$ are introduced,

$$
\mathcal{P}_{j_{1}}: \boldsymbol{z}^{j_{1}, \mathrm{opt}}=\arg \max _{\boldsymbol{z}^{j_{1}} \in \mathcal{C}_{\mathrm{ad}}^{j_{1}}} \mathcal{L}_{j_{1}}\left(\boldsymbol{z}^{j_{1}}\right)
$$




$$
\mathcal{C}_{\mathrm{ad}}^{j_{1}}=\left\{\boldsymbol{z}^{j_{1}} \in \mathbb{R}^{N},\left\|\boldsymbol{z}^{j_{1}}\right\|=1\right\}
$$

and for $k=2, \ldots, n$,

$$
\begin{gathered}
\mathcal{P}_{j_{k}}: \boldsymbol{z}^{j_{k}, \mathrm{opt}}=\arg \max _{\boldsymbol{z}^{j_{k} \in \mathcal{C}_{\mathrm{ad}}^{j_{k}}}} \mathcal{L}_{j_{k}}\left(\boldsymbol{z}^{j_{k}}\right) \\
\mathcal{C}_{\mathrm{ad}}^{j_{k}}=\left\{\boldsymbol{z}^{j_{k}} \in \mathbb{R}^{N},<\boldsymbol{z}^{j_{1}, \mathrm{opt}}, \boldsymbol{z}^{j_{k}}>=\ldots=<\boldsymbol{z}^{j_{k-1}, \mathrm{opt}}, \boldsymbol{z}^{j_{k}}>=0,\left\|\boldsymbol{z}^{j_{k}}\right\|=1\right\},
\end{gathered}
$$

in which, for $k=1, \ldots, n$, the log-likelihood function $\mathcal{L}_{j_{k}}\left(\boldsymbol{z}^{j_{k}}\right)$ is defined by

$$
\mathcal{L}_{j_{k}}\left(\boldsymbol{z}^{j_{k}}\right)=\sum_{\ell=1}^{\nu_{\mathrm{KL}}} \log _{10} p_{\eta_{j_{k}}^{\text {chaos }}(N)}\left(\eta_{j_{k}}^{\text {prior }}\left(\theta_{\ell}\right) ; \boldsymbol{z}^{j_{k}}\right)
$$

For given $\left\{j_{1}, \ldots, j_{n}\right\}$ in $\mathcal{J}$, the optimal solution $\left(\boldsymbol{z}^{j_{1}, \mathrm{opt}}, \ldots, \boldsymbol{z}^{j_{n}, \text { opt }}\right)$ yields the value $\mathcal{L}^{\text {opt }}\left(j_{1}, \ldots, j_{n}\right)=\sum_{k=1}^{n} \mathcal{L}_{j_{k}}\left(\boldsymbol{z}^{j_{k} \text {,opt }}\right)$ of the log-likelihood function. Therefore, the solution of the optimization problem defined by Eqs. (33) to (35) is given by $\left(\boldsymbol{z}^{j_{1}^{\text {opt }}, \text { opt }}, \ldots, \boldsymbol{z}^{j_{n}^{\text {opt }}, \text { opt }}\right)$ corresponding to the permutation $\left\{j_{1}^{\text {opt }}, \ldots, j_{n}^{\text {opt }}\right\}=$ $\arg \max _{\left\{j_{1}, \ldots, j_{n}\right\} \in \mathcal{J}} \mathcal{L}^{\text {opt }}\left(j_{1}, \ldots, j_{n}\right)$.

\subsubsection{Random search algorithm for the reformulated optimization problem}

The optimization problems which are to be solved are not convex problems. Consequently, there is no algorithm allowing to surely reach the global optimum for an a priori given CPU time. Therefore, for a given CPU time, all the possible algorithms only allow the cost function value to be improved from its initial value (the value for the initial point). For each fixed permutation $\left\{j_{1}, \ldots, j_{n}\right\}$ in $\mathcal{J}$, the $n$ recurrent optimization problems $\mathcal{P}_{j_{1}}, \ldots, \mathcal{P}_{j_{n}}$ are then solved with an adapted random search algorithm which allows the initial value to be improved. The quality of the approximation constructed is proportional to the CPU time used. Larger is the CPU time used, better is the approximation constructed.

- Optimization problem $\mathcal{P}_{j_{1}}$ defined by Eqs. (36) to (37)

Let $\boldsymbol{Z}_{0}^{j_{1}}=\left(Z_{0,1}^{j_{1}}, \ldots, Z_{0, N}^{j_{1}}\right)$ be a $\mathbb{R}^{N}$-valued random variable for which the components are mutually independent. Let $\boldsymbol{Z}^{j_{1}}=\boldsymbol{Z}_{0}^{j_{1}} /\left\|\boldsymbol{Z}_{0}^{j_{1}}\right\|$. Consequently, any realization $\boldsymbol{z}^{j_{1}}=\left(z_{1}^{j_{1}}, \ldots, z_{N}^{j_{1}}\right)$ of $\boldsymbol{Z}^{j_{1}}$ belongs to the admissible set $\mathcal{C}_{\text {ad }}^{j_{1}}$ defined by Eq. (37). For computing $\mathcal{L}_{j_{1}}\left(\boldsymbol{z}^{j_{1}}\right)$ defined by Eq. (40), the values of the probability density function $p_{\eta_{j_{1}}^{\text {chas }}(N)}\left(\eta_{j_{1}}^{\text {prior }}\left(\theta_{\ell}\right) ; \boldsymbol{z}^{j_{1}}\right)$ of the random variable $\eta_{j_{1}}^{\text {chaos }}(N)=\sum_{\alpha=1}^{N} z_{\alpha}^{j_{1}} \Psi_{\alpha}(\boldsymbol{\Xi})$ is calculated using the Monte Carlo simulation and 
the kernel density estimation method [4] with $\nu_{\text {chas }}$ independent realizations of normalized Gaussian vector $\Xi$ and with the $\nu_{\mathrm{KL}}$ independent realizations of the prior model $\eta_{j_{1}}^{\text {prior }}\left(\theta_{\ell}\right)$. A random search algorithm (Latin hypercube sampling type) is performed in two steps. For the first step, each component $Z_{0, \alpha}^{j_{1}}$ of random vector $Z_{0}^{j_{1}}$ is uniformly distributed on $[-1,1]$. The first step consists in $\nu_{j_{1}}$ realizations of $Z_{0}^{j_{1}}$ allowing a first approximation $\boldsymbol{z}^{j_{1}, \mathrm{opt}_{0}}$ of Eq. (36) to be carried out and corresponding to the optimal value $\boldsymbol{z}_{0}^{j_{1}, \mathrm{opt}_{0}}$ of $\boldsymbol{z}_{0}^{j_{1}}$. For the second step, each component $Z_{0, \alpha}^{j_{1}}$ of random vector $Z_{0}^{j_{1}}$ is uniformly distributed on $\left[z_{0, \alpha}^{j_{1}, \mathrm{opt}_{0}}-\delta_{\alpha}, z_{0, \alpha}^{j_{1}, \mathrm{opt}_{0}}+\delta_{\alpha}\right]$ with $\delta_{\alpha}=\left(1-z_{0, \alpha}^{j_{1}, \text { opt }_{0}}\right) / 10$. The second step consists in $\nu_{j_{1}}$ realizations of $\boldsymbol{Z}_{0}^{j_{1}}$ allowing a final approximation $\boldsymbol{z}^{j_{1} \text { opt }}$ of Eq. (36) to be carried out.

- Optimization problem $\mathcal{P}_{j_{k}}$ for $k=2, \ldots, n$ defined by Eqs. (38) to (39) For fixed $k$, the optimal vectors $\boldsymbol{z}^{j_{1} \text {,opt }}, \ldots, \boldsymbol{z}^{j_{k-1}, \text { opt }}$ in $\mathbb{R}^{N}$ are known from $\mathcal{P}_{j_{1}}$, $\ldots, \mathcal{P}_{j_{k-1}}$. Let $[H]$ be the $(N \times(k-1))$ real matrix whose columns are the vectors $\boldsymbol{z}^{j_{1}, \mathrm{opt}}, \ldots, \boldsymbol{z}^{j_{k-1}, \mathrm{opt}}$. Let $\boldsymbol{b}^{1}, \ldots, \boldsymbol{b}^{d}$ with $d=N-k+1$ be an orthonormal basis of the null space of $[H]^{T}$. Let $\boldsymbol{q}_{0}=\left(q_{0,1}, \ldots, q_{0, d}\right)$ be any vector in $\mathbb{R}^{d}$ and let $\boldsymbol{q}=\boldsymbol{q}_{0} /\left\|\boldsymbol{q}_{0}\right\|$ be the corresponding vector in $\mathbb{R}^{d}$ for which the Euclidean norm equal to 1 . Then, the vector $\boldsymbol{z}^{j_{k}}=q_{1} \boldsymbol{b}^{1}+\ldots+q_{d} \boldsymbol{b}^{d}$ in $\mathbb{R}^{N}$ has an Euclidean norm equal to 1 and is orthogonal to the vectors $\boldsymbol{z}^{j_{1}, \mathrm{opt}}, \ldots, \boldsymbol{z}^{j_{k-1}, \mathrm{opt}}$. We then conclude that such a vector $\boldsymbol{z}^{j_{k}}$ belongs to the admissible set $\mathcal{C}_{\text {ad }}^{j_{k}}$ defined by Eq. (39). Let $\boldsymbol{Q}_{0}=\left(Q_{0,1}, \ldots, Q_{0, d}\right)$ be a $\mathbb{R}^{d}$-valued random variable for which the components are mutually independent. Let $Q=Q_{0} /\left\|Q_{0}\right\|$. Consequently, any realization $\boldsymbol{z}^{j_{k}}=\left(z_{1}^{j_{k}}, \ldots, z_{N}^{j_{k}}\right)$ of $\boldsymbol{Z}^{j_{k}}=Q_{1} \boldsymbol{b}^{1}+\ldots+Q_{d} \boldsymbol{b}^{d}$ belongs to the admissible set $\mathcal{C}_{\text {ad }}^{j_{k}}$. For computing $\mathcal{L}_{j_{k}}\left(\boldsymbol{z}^{j_{k}}\right)$ defined by Eq. (40), the values of the probability density function $p_{\eta_{j_{k}}^{\text {chaos }}(N)}\left(\eta_{j_{k}}^{\text {prior }}\left(\theta_{\ell}\right) ; \boldsymbol{z}^{j_{k}}\right)$ of the random variable $\eta_{j_{k}}^{\text {chaos }}(N)=\sum_{\alpha=1}^{N} z_{\alpha}^{j_{k}} \Psi_{\alpha}(\boldsymbol{\Xi})$ is calculated using Monte Carlo simulation and the kernel density estimation method with $\nu_{\text {chaos }}$ independent realizations of normalized Gaussian vector $\Xi$ and with the $\nu_{\mathrm{KL}}$ independent realizations of the prior model $\eta_{j_{k}}^{\text {prior }}\left(\theta_{\ell}\right)$. As for the optimization problem $\mathcal{P}_{j_{1}}$, a similar random search algorithm is performed in two steps. For the first step, each component $Q_{0, i}$ of random vector $\boldsymbol{Q}_{0}$ is uniformly distributed on $[-1,1]$. The first step consists in $\nu_{j_{k}}$ realizations of $\boldsymbol{Q}_{0}$ allowing a first approximation $\boldsymbol{z}^{j_{k}, \mathrm{opt}_{0}}$ of Eq. (38) to be carried out and corresponding to the optimal value $\boldsymbol{q}_{0}^{\mathrm{opt}_{0}}$ of $\boldsymbol{q}_{0}$. For the second step, each component $Q_{0, i}$ of random vector $\boldsymbol{Q}_{0}$ is uniformly distributed on $\left[q_{0, i}^{\mathrm{opt}_{0}}-\delta_{i}, q_{0, i}^{\mathrm{opt}_{0}}+\delta_{i}\right]$ with $\delta_{i}=\left(1-q_{0, i}^{\mathrm{opt}_{0}}\right) / 10$. The second step consists in $\nu_{j_{k}}$

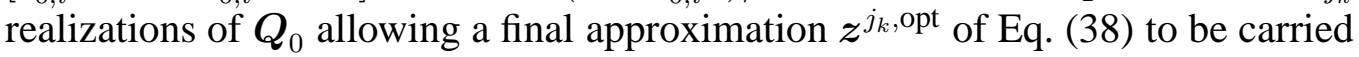


out.

\subsection{Algorithm for identification of the random coefficients of the polynomial chaos expansion (Step5)}

An adapted algorithm is proposed to construct $\underline{\mathcal{Y}}^{\text {opt }}=\left(\underline{\boldsymbol{y}}^{1, \mathrm{opt}}, \ldots, \underline{\boldsymbol{y}}^{N, \mathrm{opt}}\right)$ and $\boldsymbol{\sigma}^{\mathrm{opt}}=\left(\sigma_{1}^{\mathrm{opt}}, \ldots, \sigma_{N}^{\mathrm{opt}}\right)$ from the optimization problem defined by Eq. (30) with Eq. (31). This algorithm is based on a trial search method with a very low number of unknown parameters (two scalar parameters).

Firstly, the solution $\underline{\mathcal{Y}}^{\mathrm{opt}}$ in $\underline{\mathcal{Y}}$ is searched in a neighborhood of the optimal deterministic coefficients $\mathcal{Y}^{\text {opt }}=\left(\boldsymbol{y}^{1, \mathrm{opt}}, \ldots, \boldsymbol{y}^{N \text {,opt }}\right)$ of the polynomial expansion of the prior model, computed in Step 4. The diameter is defined by a given parameter $\varepsilon_{1}$ such that $0<\varepsilon_{1}$ and we choose $\underline{\mathcal{Y}}=\varepsilon_{1} \mathcal{Y}^{\text {opt }}$ (if $\varepsilon_{1}=1$ then $\underline{\mathcal{Y}}^{\text {opt }}=\mathcal{Y}^{\text {opt }}$ ). Consequently, the optimal solution can be written as $\underline{\mathcal{Y}}^{\text {opt }}=\varepsilon_{1}^{\text {opt }} \mathcal{Y}^{\text {opt }}$ in which $\varepsilon_{1}^{\text {opt }}$ is the optimal value of the scalar parameter $\varepsilon_{1}$.

Secondly, $\boldsymbol{\sigma}=\left(\sigma_{1}, \ldots, \sigma_{N}\right)$ is replaced by $\boldsymbol{\sigma}=\varepsilon_{2} \mathbf{1}$ in which $\mathbf{1}=(1, \ldots, 1)$ that is to say $\sigma_{1}=\ldots=\sigma_{N}=\varepsilon_{2}$. This means that the $N$ parameters $\sigma_{1}, \ldots, \sigma_{N}$ are replaced by only one parameter $\varepsilon_{2} \geq 0$ in which the parameter $\varepsilon_{2}$ allows the variance to be controlled. If $\varepsilon_{2}=0$, then $\boldsymbol{Y}^{\alpha}=\boldsymbol{y}^{\alpha}$.

We then conclude that the optimization problem defined by Eq. (30) can be rewritten as

$$
\left(\varepsilon_{1}^{\text {opt }}, \varepsilon_{2}^{\text {opt }}\right)=\arg \min _{\varepsilon_{1}>0, \varepsilon_{2} \geq 0} \mathcal{J}^{\text {post }}\left(\varepsilon_{1} \mathcal{Y}^{\text {opt }}, \varepsilon_{2} \mathbf{1}\right)
$$

The optimization problem defined by Eq. (41) can be solved, for instance, using the trial method.

\section{Application}

(1) Definition of the stochastic boundary value problem at the meso-scale. We consider a microstructure represented by an open bounded domain $\Omega=(] 0,1[)^{3}$ of $\mathbb{R}^{3}$ with generic point $\boldsymbol{x}=\left(x_{1}, x_{2}, x_{3}\right)$ for which the origine $(0,0,0)$ is located at a corner of the cube (see Fig. 1 left). Domain $\Omega$ is occupied by a heterogeneous complex material modeled by a statistically homogeneous and anisotropic elastic random medium at the meso-scale. For this meso-scale modeling, the elastic properties of the microstructure are then defined by the non-Gaussian fourth-order tensor-valued random field $\{\boldsymbol{C}(\boldsymbol{x}), \boldsymbol{x} \in \Omega\}$ in which $\boldsymbol{C}(\boldsymbol{x})=\left\{\boldsymbol{C}_{i j k \ell}(\boldsymbol{x})\right\}_{i j k \ell}$. Let $\left\{\boldsymbol{u}(\boldsymbol{x})=\left(u_{1}(\boldsymbol{x}), u_{2}(\boldsymbol{x}), u_{3}(\boldsymbol{x})\right), \boldsymbol{x} \in \Omega\right\}$ be the displacement random field at 
the meso-scale. The random constitutive equation is then written as $\boldsymbol{s}_{j k}(\boldsymbol{x})=$ $\boldsymbol{C}_{j k \ell m}(\boldsymbol{x}) \boldsymbol{\varepsilon}_{\ell m}(\boldsymbol{x})$, in which $\boldsymbol{s}$ is the stress tensor and $\boldsymbol{\varepsilon}$ is the strain tensor such that $\varepsilon_{\ell m}(\boldsymbol{u}(\boldsymbol{x}))=\frac{1}{2}\left(\partial u_{\ell}(\boldsymbol{x}) / \partial x_{m}+\partial u_{m}(\boldsymbol{x}) / \partial x_{\ell}\right)$. The boundary $\partial \Omega$ is written as $\Gamma_{0} \cup \Gamma_{\text {obs }} \cup \Gamma$. A Dirichlet condition $\boldsymbol{u}=0$ is given on $\Gamma_{0}$ while a Neumann condition is given on $\Gamma$ corresponding to the application of a given deterministic surface force field $\boldsymbol{g}^{\Gamma}(\boldsymbol{x})=\left(g_{1}^{\Gamma}(\boldsymbol{x}), g_{2}^{\Gamma}(\boldsymbol{x}), g_{3}^{\Gamma}(\boldsymbol{x})\right)$. There is no surface force field applied to $\Gamma_{\text {obs }}$ which is the part of the boundary for which field $\boldsymbol{u}$ is observed (this corresponds to the hypothesis for which only partial experimental data are observed and then are available). The stochastic boundary value problem consists in finding the second-order random field $\{\boldsymbol{u}(\boldsymbol{x}), \boldsymbol{x} \in \Omega\}$ such that

$$
\begin{array}{rll}
-\operatorname{div} \boldsymbol{s}=0 & \text { in } & \Omega, \\
\boldsymbol{u}(\boldsymbol{x})=\mathbf{0} & \text { on } & \Gamma_{0}, \\
\boldsymbol{s}(\boldsymbol{x}) \boldsymbol{n}(\boldsymbol{x})=\boldsymbol{g}^{\Gamma}(\boldsymbol{x}) \quad \text { on } \quad \Gamma & \text { and } & \boldsymbol{s}(\boldsymbol{x}) \boldsymbol{n}(\boldsymbol{x})=\mathbf{0} \quad \text { on } \Gamma_{\text {obs }},
\end{array}
$$

in which $\boldsymbol{n}(\boldsymbol{x})=\left(n_{1}(\boldsymbol{x}), n_{2}(\boldsymbol{x}), n_{3}(\boldsymbol{x})\right)$ is the outward unit normal to $\partial \Omega$, where $\{\operatorname{div} \boldsymbol{s}(\boldsymbol{x})\}_{j}=\partial s_{j k}(\boldsymbol{x}) / \partial x_{k}$ and where the random constitutive equation is defined above.

(2) Family of prior probability models for the random field $\{\boldsymbol{C}(\boldsymbol{x}), \boldsymbol{x} \in \Omega\}$. The stochastic boundary value problem defined by Eq. (42) is elliptic. The family $\left\{\boldsymbol{C}^{\text {prior }}(\boldsymbol{x} ; \boldsymbol{w}), \boldsymbol{x} \in \Omega\right\}$ of the prior probability models for the fourth-order tensor-valued random field $\{\boldsymbol{C}(\boldsymbol{x}), \boldsymbol{x} \in \Omega\}$ could arbitrarily be chosen (see for instance [2, 3, 63] for the scalar case). In this application, we are interested in the anisotropic case that is to say to the tensor case. We then choose the probabilistic model constructed in $[50,51]$ ) using the Maximum Entropy Principle under the constraints defined by the available information and yielding a stochastic non-uniform ellipticity condition. All the details concerning the construction and the generation of independent realizations for such a non-Gaussian fourth-order tensor-valued random field can be found in [50, 51]). For all $\boldsymbol{x}$ in $\Omega$, the fourth-order tensor-valued random field $C^{\text {prior }}(\boldsymbol{x} ; \boldsymbol{w})$ is represented by the symmetric positive-definite $(6 \times 6)$ real random matrix $\left[\boldsymbol{A}^{\text {prior }}(\boldsymbol{x} ; \boldsymbol{w})\right]$ such that $\left[\boldsymbol{A}^{\text {prior }}(\boldsymbol{x} ; \boldsymbol{w})\right]_{I J}=\boldsymbol{C}_{i j k \ell}^{\text {prior }}(\boldsymbol{x} ; \boldsymbol{w})$. This family of prior models $\left\{\left[\boldsymbol{A}^{\text {prior }}(\boldsymbol{x}, \boldsymbol{w})\right], \boldsymbol{x} \in\right.$ $\Omega\}$ depends on the vector valued parameters $\boldsymbol{w}=\left(\delta, L,\left\{[\underline{A}]_{i j}, 1 \leq i \leq j \leq 6\right\}\right)$ belonging to an admissible subset of $\mathbb{R}^{23}$ for which $\delta>0$ is a real parameter controlling the level of statistical fluctuations of the field, $L$ is a correlation length (with respect to the general model presented in $[50,51]$, it is assumed that the three spatial correlation lengths $L_{1}, L_{2}$ and $L_{3}$ are equal; we then have 
$\left.L=L_{1}=L_{2}=L_{3}\right)$. The mean value $E\left\{\left[\boldsymbol{A}^{\text {prior }}(\boldsymbol{x} ; \boldsymbol{w})\right]\right\}=[\underline{A}]$ (which is independent of $\boldsymbol{x}$ because $\left\{\left[\boldsymbol{A}^{\text {prior }}(\boldsymbol{x} ; \boldsymbol{w})\right], \boldsymbol{x} \in \Omega\right\}$ is the restriction to $\Omega$ of a homogeneous random field) is a symmetric positive-definite $(6 \times 6)$ real matrix for which 21 parameters must be identified for an anisotropic material.

(3) Stochastic finite element approximation of the stochastic boundary value problem. The cube (] $0,1[)^{3}$ is meshed with $6 \times 6 \times 6=216$ finite elements using 8 -nodes finite elements (see Fig. 1 left). There are 8 integration points in each finite element. Since there are 216 finite elements, we have $N_{p}=1728$ integration points. The dimension of vector $\mathbb{V}$ discretizing $[\boldsymbol{A}()$.$] (or vector \mathbb{V}^{\text {prior }}(\boldsymbol{w})$ discretizing $\left.\left[\boldsymbol{A}^{\text {prior }}(. ; \boldsymbol{w})\right]\right)$ is then $m_{\mathbb{V}}=21 \times N_{p}=36288$. It should be noted that the number of independent Gaussian germs used to generate such a stochastic model is then 36288 . Concerning the boundary conditions, the displacements are locked at points $(1,0,0),(1,1,0),(1,1,1)$ and $(1,0,1)$ corresponding to the 4 corners of the face of the cube in the plane $x_{1}=1$. An external point load $(0,1,0)$ is applied to the node of coordinates $(0,0,1)$. The observed degrees of freedom are the $x_{2}$ - and $x_{3}$-displacements of the nodes located inside the face $x_{1}=0$. Since there are 49 nodes on each face of the cube whose 25 nodes inside the face, there are $m=2 \times 25=50$ observed degrees of freedom.
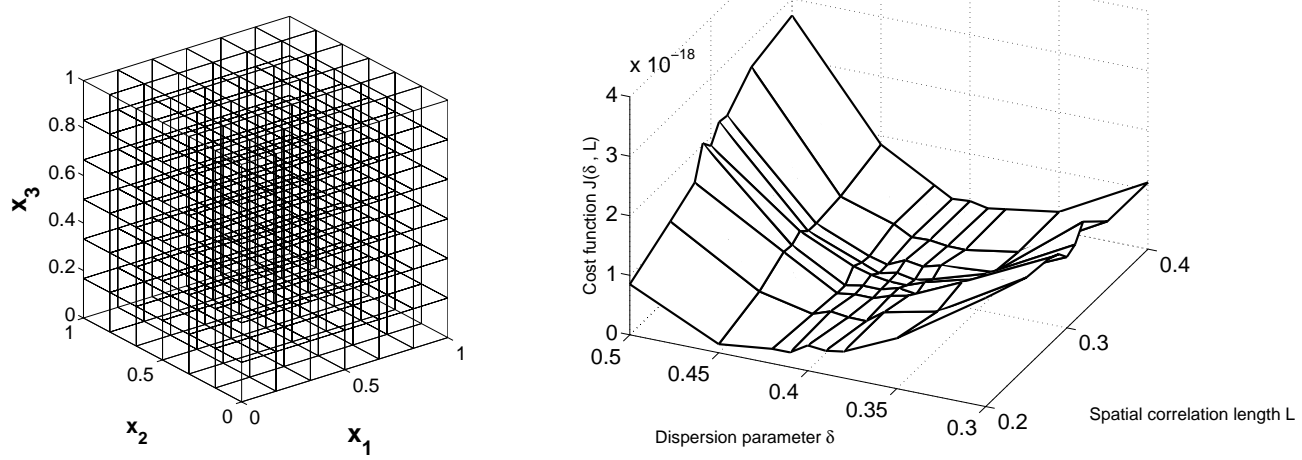

Figure 1: (Left figure): Finite element mesh of the domain. (Right figure): Cost function $(\delta, L) \mapsto$ $J\left(\delta, L,\left\{[\underline{A}]_{i j}, 1 \leq i \leq j \leq 6\right\}\right)$ for the identification of the optimal prior probability model using the experimental data sets.

(4) Experimental data sets. The objective of this application is to validate the methodology proposed for partial experimental data (in this application, the ob- 
servation vector is only made of 50 degrees of freedom among a total of 1017 degrees of freedom). The experimental data are then synthetically generated using the stochastic boundary value problem with a perturbed version of the prior model. We then consider $\nu_{\exp }=110$ experimental data sets $\boldsymbol{u}^{\exp , 1}, \ldots, \boldsymbol{u}^{\exp , \nu_{\exp }}$. Each experimental data set is defined as the observation vector $\boldsymbol{u}^{\exp , \ell}$ in $\mathbb{R}^{m}$ corresponding to an independent realization of the response of the stochastic boundary value problem that is to say, is such that $\boldsymbol{u}^{\exp , \ell}=\boldsymbol{h}\left(\mathbb{V}^{\exp }\left(\theta_{\ell}\right)\right)$. The random vector $\mathbb{V}^{\exp }=\mathbb{V}_{\text {pert }}^{\text {prior }}\left(\boldsymbol{w}^{\exp }\right)$ is a perturbation of the probabilistic model of the prior model $\mathbb{V}^{\text {prior }}(\boldsymbol{w})$. For this perturbation of the probabilistic model, the mean matrix $\left[\underline{A}^{\exp }\right]$ is not modified. The parameter $\boldsymbol{w}=\boldsymbol{w}^{\exp }=\left(\delta^{\exp }, L^{\exp },\left\{\left[\underline{A}^{\exp }\right]_{i j}, 1 \leq i \leq j \leq\right.\right.$ $6\})$ with $\delta^{\exp }=0.37, L^{\exp }=0.33$ and where the $(6 \times 6)$ real matrix $\left[\underline{A}^{\text {exp }}\right]$ of the mean model for the anisotropic material is such that

$$
\left[\underline{A}^{\exp }\right]=10^{10} \times\left[\begin{array}{cccccc}
3.3617 & 1.7027 & 1.3637 & -0.1049 & -0.2278 & 2.1013 \\
1.7027 & 1.6092 & 0.7262 & 0.0437 & -0.1197 & 0.8612 \\
1.3637 & 0.7262 & 1.4653 & -0.1174 & -0.1506 & 1.0587 \\
-0.1049 & 0.0437 & -0.1174 & 0.1319 & 0.0093 & -0.1574 \\
-0.2278 & -0.1197 & -0.1506 & 0.0093 & 0.1530 & -0.1303 \\
2.1013 & 0.8612 & 1.0587 & -0.1574 & -0.1303 & 1.7446
\end{array}\right] .
$$

The matrix defined by Eq. (43) corresponds to the mean value of the elasticity tensor of the anisotropic random microstructure presented in [51].

(5) Identification with experimental data of an optimal prior model in the constructed family. The optimization problem defined by Eq. (13) related to the identification of the optimal prior probability model using the experimental data is solved by the trial method. For the parameter $\boldsymbol{w}=\left(\delta, L,\left\{[\underline{A}]_{i j}, 1 \leq i \leq j \leq\right.\right.$ $6\}$ ), only the two parameters $\delta$ and $L$ are free in the admissible set and $[\underline{A}]$ is fixed to the value $\left[\underline{A}^{\exp }\right]$. For the trial method, the cost function $\boldsymbol{w} \mapsto J(\boldsymbol{w})$ is computed for $\delta \in\{0.3,0.35,0.38,0.39,0.4,0.41,0.42,0.45,0.5\}$, for $L \in$ $\{0.2,0.25,0.28,0.29,0.3,0.31,0.32,0.35,0.4\}$ and for $[\underline{A}]=\left[\underline{A}^{\exp }\right]$. For each trial point $\boldsymbol{w}$, the value of the cost function $J^{\text {prior }}(\boldsymbol{w})$ is estimated using the stochastic numerical model $\boldsymbol{U}^{\text {prior }}(\boldsymbol{w})=\boldsymbol{h}\left(\mathbb{V}^{\text {prior }}(\boldsymbol{w})\right)$ which is solved by the Monte Carlo method with 500 independent realizations of random vector $\mathbb{V}^{\text {prior }}(\boldsymbol{w})$. The optimal value $\boldsymbol{w}^{\mathrm{opt}}=\left(\delta^{\mathrm{opt}}, L^{\mathrm{opt}},\left\{[\underline{A}]_{i j}, 1 \leq i \leq j \leq 6\right\}\right)$ corresponding to the minimum of the cost function is obtained for $\delta^{\mathrm{opt}}=0.4$ and $L^{\mathrm{opt}}=0.3$. Fig. 1 right displays the graph of the cost function $(\delta, L) \mapsto J\left(\delta, L,\left\{\left[\underline{A}_{i j}, 1 \leq i \leq j \leq 6\right\}\right)\right.$. 
(6) Construction of the statistical reduced order optimal prior model. The optimal prior model $\mathbb{V}^{\text {prior }}\left(\boldsymbol{w}^{\text {opt }}\right)$ is simply denoted by $\mathbb{V}^{\text {prior }}$. Its mean value $\underline{\mathbb{V}}=E\left\{\mathbb{V}^{\text {prior }}\left(\boldsymbol{w}^{\mathrm{opt}}\right)\right\}$ is a vector in $\mathbb{R}^{36288}$ and its covariance matrix $\left[C_{\text {Vprior }}\right]$ is a $(36288 \times 36288)$ real symmetric matrix. These two second-order moments are estimated with the optimal prior model using $\nu_{\mathrm{KL}}=1000$ independent realizations. The dominant eigenspace of the eigenvalue problem $\left[C_{\text {Vprior }}\right] \mathbb{W}^{j}=\lambda_{j} \mathbb{W}^{j}$ is solved by using the usual subspace iteration method without assembling matrix $\left[C_{\text {Vprior }}\right]$. The $(36288 \times n)$ real matrix $[\mathbb{W}]=\left[\mathbb{W}^{1} \ldots \mathbb{W}^{n}\right]$ of the $n$ eigenvectors associated with the $n$ largest eigenvalues $\lambda_{1} \geq \lambda_{2} \geq \ldots \geq \lambda_{n}>0$ is such that $[\mathbb{W}]^{T}[\mathbb{W}]=\left[I_{n}\right]$. Fig. 2 left displays the graph of the relative error function $n \mapsto \operatorname{err}(n)=1-\frac{\sum_{j=1}^{n} \lambda_{j}}{\operatorname{tr}\left[C_{\text {vprior }}\right]}$ related to the convergence (with respect to $n$ ) of the expansion of random vector $\mathbb{V}^{\text {prior }}$ corresponding to the optimal prior model. The left figure shows that a reasonable convergence is reached for $n=550$. Fig. 2 right displays the distribution of the values of the first 550 largest eigenvalues of covariance matrix $\left[C_{\text {Vprior }}\right]$.
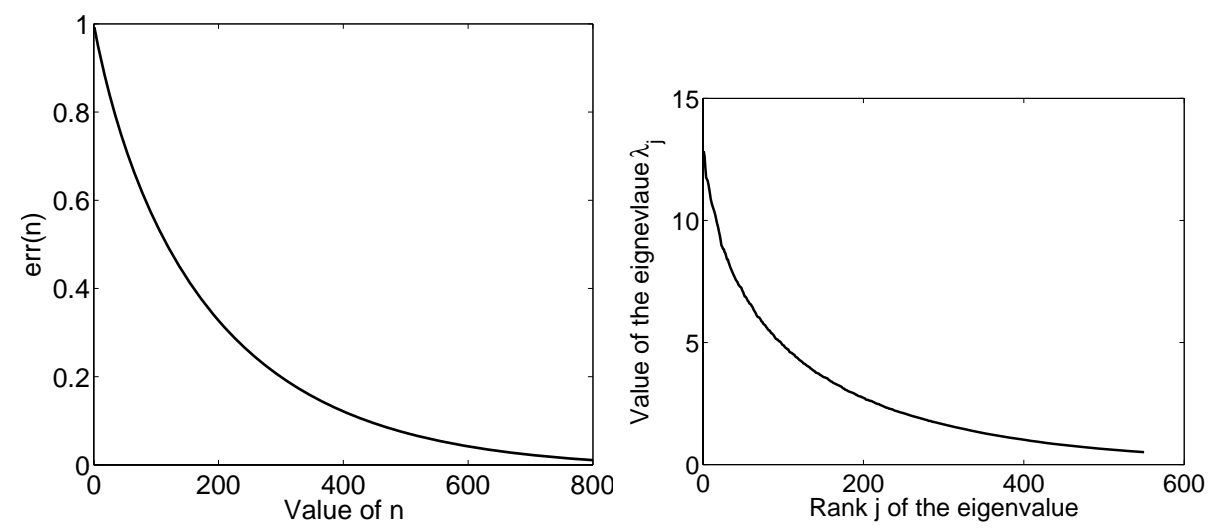

Figure 2: (Left figure): Graph of the error function $n \mapsto \operatorname{err}(n)$. (Right figure): Distribution of the first 550 largest eigenvalues of covariance matrix $\left[C_{\mathbb{V} \text { prior }}\right]$.

(7) Construction of the polynomial chaos expansion with deterministic vectorvalued coefficients of the reduced-order optimal prior model. The calculation of the optimal values of the deterministic vector-valued coefficients $\boldsymbol{y}^{1}, \ldots, \boldsymbol{y}^{N}$ in $\mathbb{R}^{n}$ of the polynomial chaos expansion $\boldsymbol{\eta}^{\text {chaos }}(N)=\sum_{\alpha=1}^{N} \boldsymbol{y}^{\alpha} \Psi_{\alpha}(\boldsymbol{\Xi})$ is performed using the random search algorithm presented in Section 3.1 with $n=550$ (which means that each unknown vector $\boldsymbol{y}^{\alpha}$ is in $\mathbb{R}^{550}$ ). This calculation is carried out for the optimal prior model and for a germ $\Xi=\left(\xi_{1}, \ldots, \xi_{N_{g}}\right)$ of length 
$N_{g}$. In the random search algorithm, only one permutation in $\mathcal{I}$ is used and is $\left\{j_{1}=1, \ldots, j_{n}=n\right\}$ that is to say $\{1, \ldots, n\}$. Each recurrent optimization problem $\mathcal{P}_{j}$, for $j=1, \ldots, 550$, is solved using $\nu_{j}$ independent realizations. Fig. 3 left displays the graph of function $j \mapsto \nu_{j}$. The total number of independent realizations performed with the algorithm is then $2 \times \sum_{j=1}^{550} \nu_{j}=27372$.

For a fixed value of $N_{g}$ and for a fixed maximum degree $N_{d}$ of the polyno-
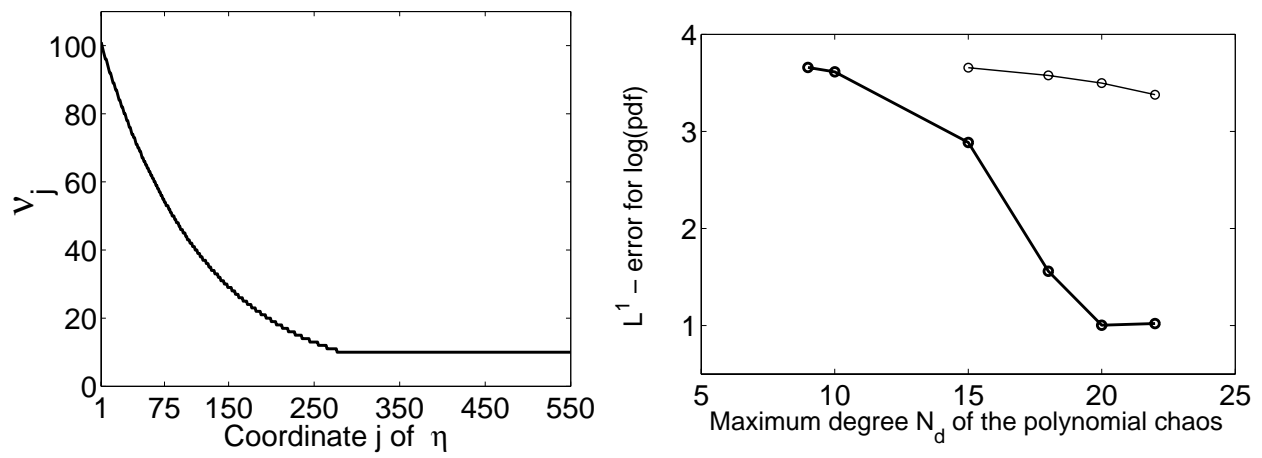

Figure 3: (Left figure): Graph of function $j \mapsto \nu_{j}$. (Right figure): $L^{1}$-error function $N_{d} \mapsto$ $\operatorname{err}\left(N_{g}, N_{d}\right)$ for random vector $\eta^{\text {chaos }}(N)$ with $N=h\left(N_{g}, N_{d}\right)$ and for $N_{g}=3$ (thin line) and $N_{g}=4$ (thick line).

mial chaos, the polynomial chaos expansion with deterministic vector-valued coefficients for the optimal prior model has been carried with $N$ chaos (that is to say, the number of deterministic vector-valued coefficients $\boldsymbol{y}^{1}, \ldots, \boldsymbol{y}^{N}$ in $\mathbb{R}^{550}$ is $N=h\left(N_{g}, N_{d}\right)=\left(N_{d}+N_{g}\right) ! /\left(N_{d} ! N_{g} !\right)-1$. In order to calculate the loglikelihood function, the probability density function $e \mapsto p_{\eta_{j}^{\text {chaos }}(N)}\left(e ; \boldsymbol{y}^{1}, \ldots, \boldsymbol{y}^{N}\right)$ of $\eta_{j}^{\text {chaos }}(N)$ such that $\eta_{j}^{\text {chaos }}(N)=\sum_{\alpha=1}^{N} y_{j}^{\alpha} \Psi_{\alpha}(\boldsymbol{\Xi})$ is estimated for the $\nu_{\mathrm{KL}}=$ 1000 independent realizations $\eta_{j}^{\text {prior }}\left(\theta_{\ell}\right)$ used previously for $\ell=1, \ldots, 1000$. The values of the probability density function $p_{\eta_{j}^{\text {chaos }}(N)}\left(\eta_{j}^{\text {prior }}\left(\theta_{\ell}\right) ; \boldsymbol{y}^{1}, \ldots, \boldsymbol{y}^{N}\right)$ is estimated using the kernel density estimation method and $\nu_{\text {chaos }}$ independent realizations of the normalized Gaussian vector $\boldsymbol{\Xi}$. Let $e \mapsto p_{\eta_{j}^{\text {prior }}}(e)$ be the estimation of the probability density function of the optimal prior random variable $\eta_{j}^{\text {prior }}$ also carried out with the kernel density estimation method. A convergence analysis of the polynomial chaos expansion has been performed in function of the dimension $N_{g}$ of the germ and of the maximum degree $N_{d}$ of the polynomial chaos. The polynomial chaos expansion with deterministic vector-valued coefficients of the optimal prior model has been carried out with $N_{d}=15,18,20,22$ for $N_{g}=3$ 

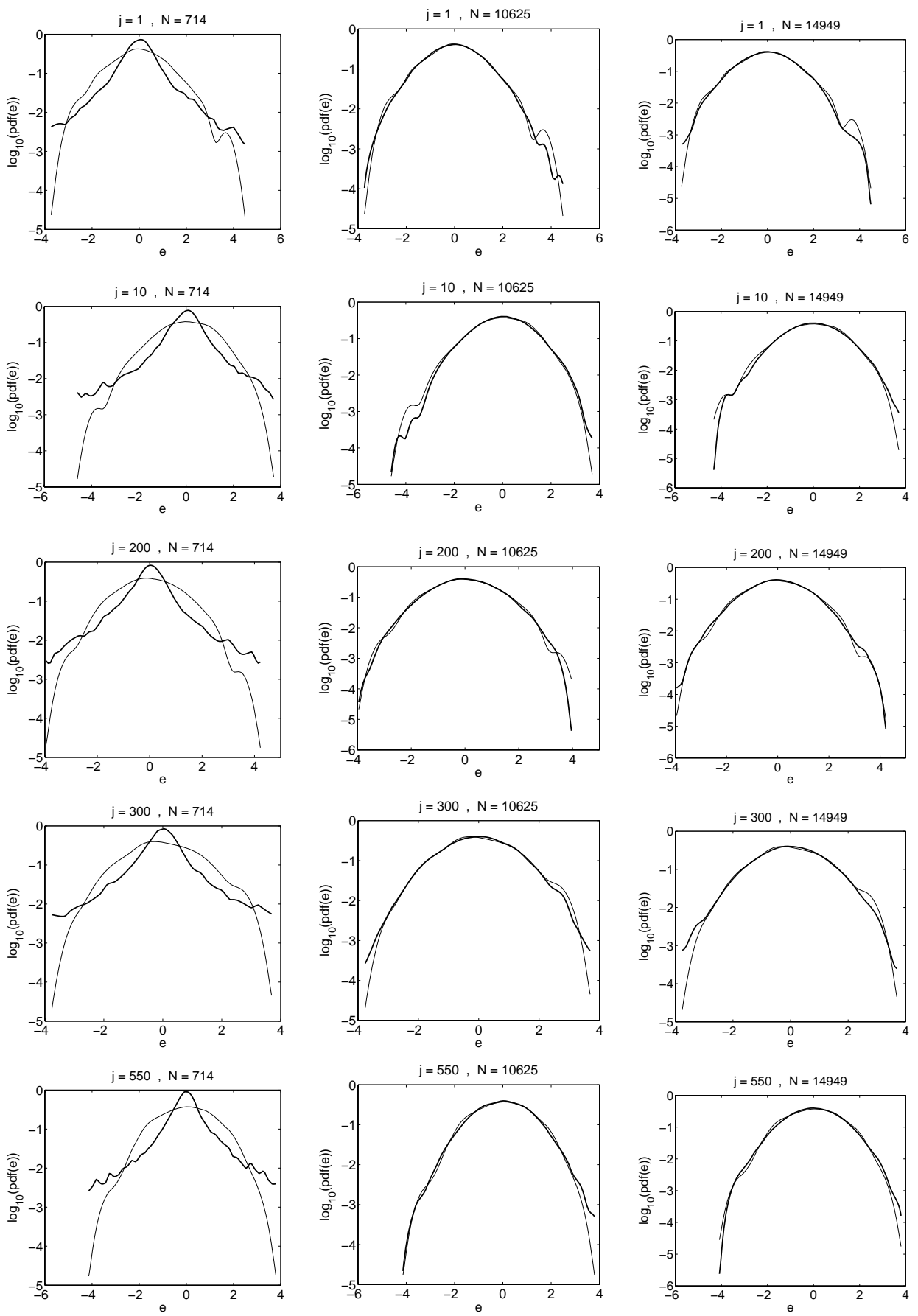

Figure 4: Convergence analysis with respect to the number $N$ of chaos. Comparisons of the graph of the optimal prior pdf $e \mapsto p_{\eta_{j}^{\text {prior }}}(e)$ (thin solid line) with the graph of the pdf $e \mapsto p_{\eta_{j}}(e)$ estimated using the polynomial chaos expansion with $N$ chaos (thick solid line), for different values of $N$ and for given coordinate $j$ (the values of $j$ and $N$ are indicated at the top of each figure). Vertical axis: $\log _{10}$ of the pdf. Horizontal axis: value e of $\eta_{j}$. 
and, with $N_{d}=9,10,15,18,20,22$ for $N_{g}=4$. The corresponding number $N$ of chaos (that is to say the number of deterministic vector-valued coefficients $\boldsymbol{y}^{1}, \ldots, \boldsymbol{y}^{N}$ in $\mathbb{R}^{550}$ ) is $N=815,1329,1770,2299$ for $N_{g}=3$ and, $N=714$, $1000,3875,7314,10625,14949$ for $N_{g}=4$. The number $\nu_{\text {chaos }}$ of independent realizations of $\boldsymbol{\Xi}$ used to estimate the probability density functions of $\eta_{j}^{\text {chaos }}(N)$ is $\nu_{\text {chaos }}=11000$ except for $N_{g}=4$ and $N_{d}=22$ for which $\nu_{\text {chaos }}=16000$. Fig. 3 right displays the graphs of the $L^{1}$-error function $N_{d} \mapsto \operatorname{err}\left(N_{g}, N_{d}\right)$ defined by Eq. (23) for $N_{g}=3$ and $N_{g}=4$. It can be seen that a reasonable convergence is reached for $N_{g}=4$ and $N_{d}=20$, that is to say for $N=N^{\text {conv }}=10625$ terms in the polynomial chaos expansion of $\boldsymbol{\eta}^{\text {chaos }}(N)$. The number $N_{g}=4$ of independent Gaussian germs with the degree $N_{d}=20$ yields $N=10625$ uncorrelated non-Gaussian (but dependent) random variables used in the polynomial chaos expansion which has to be compared to the 36288 independent Gaussian germs used to generate the non-Gaussian spatially sampled tensor-valued random field.

Fig. 4 is related to the convergence analysis with respect to the number $N=$ $h\left(N_{g}, N_{d}\right)$ of chaos. Each figure shows the comparison of the graph of the optimal prior pdf $e \mapsto \log _{10}\left(p_{\eta_{j}^{\text {prior }}}(e)\right)$ with the graph of the $\operatorname{pdf} e \mapsto \log _{10}\left(p_{\eta_{j}^{\text {chaos }}(N)}\left(e ; \boldsymbol{y}^{1, \text { opt }}\right.\right.$, $\left.\left.\ldots, \boldsymbol{y}^{N, \mathrm{opt}}\right)\right)$ estimated using the polynomial chaos expansion with $N=h\left(N_{g}, N_{d}\right)$ chaos. The figures show the comparisons for the coordinates $j=1,10,200,300$ and 550, for $N_{g}=4$ and for $N_{d}=9(N=714), N_{d}=20(N=10625)$ and for $N_{d}=22(N=14949)$. It can be seen again a good convergence of the probability density function for these five coordinates obtained for $N_{g}=4$ and $N_{d}=20$ corresponding to the value $N^{\text {conv }}=10625$ of $N$. The quality of the convergence is similar for the other 447 coordinates. Fig. 5 displays the graph of function $j \mapsto \operatorname{err}_{j}\left(N^{\text {conv }}\right)$ defined by Eq. (22) which shows the error for each coordinate $j$ and for the value of $N=N^{\text {conv }}$ for which the convergence is reached. For the configuration $10625\left(N_{g}=4, N_{d}=20\right)$ with $n=550$ and $\nu_{\text {chaos }}=11000$, the CPU time is 28 hours using one processor with one core (in fact, about 4 hours with 8 cores).

(8) Construction of the probability distribution of the random coefficients of the polynomial chaos expansion and identification of the parameters using the experimental data. The optimization problem defined by Eq. (30) is simplified using Eq. (41). The two deterministic parameters $\varepsilon_{1}>0$ and $\varepsilon_{2} \geq 0$ control the probability distribution of the random vectors $\boldsymbol{Y}^{1}, \ldots, \boldsymbol{Y}^{N}$. The optimization problem is solved using the trial method for $\varepsilon_{1}$ belonging to the set $\mathcal{E}_{1}=\{[1.000,0.920]$ with a step of -0.005$\}$ and for $\varepsilon_{2}$ belonging to the set $\mathcal{E}_{2}=\{[0.025,0.060]$ with a 


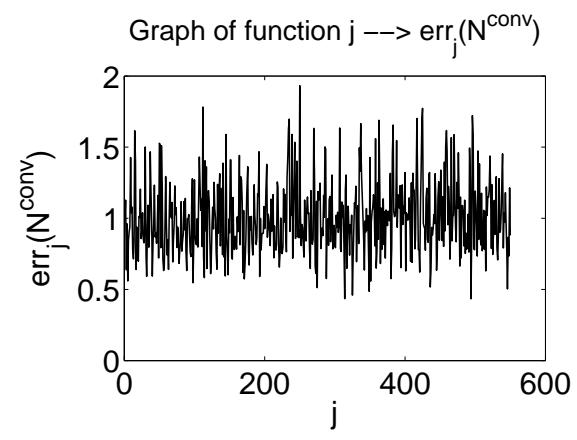

Figure 5: Graph of function $j \mapsto e \operatorname{err}_{j}\left(N^{\text {conv }}\right)$.

step of 0.005$\}$. We then introduce the mapping $j \mapsto\left(\varepsilon_{1, j}, \varepsilon_{2, j}\right)$ from $\{1, \ldots, 136\}$ into $\mathcal{E}_{1} \times \mathcal{E}_{2}$ For each fixed value of $\left(\varepsilon_{1, j}, \varepsilon_{2, j}\right)$, the stochastic boundary value problem is solved by the Monte Carlo method with $\nu_{\text {chaos }}=11,000$ independent realizations. The total CPU time for solving the optimization problem is 14 hours using 64 processors. Fig. 6 displays the graph of $j \mapsto \log _{10} \mathcal{J}^{\text {post }}\left(\varepsilon_{1, j} \mathcal{Y}^{\text {opt }}, \varepsilon_{2, j} \mathbf{1}\right)$

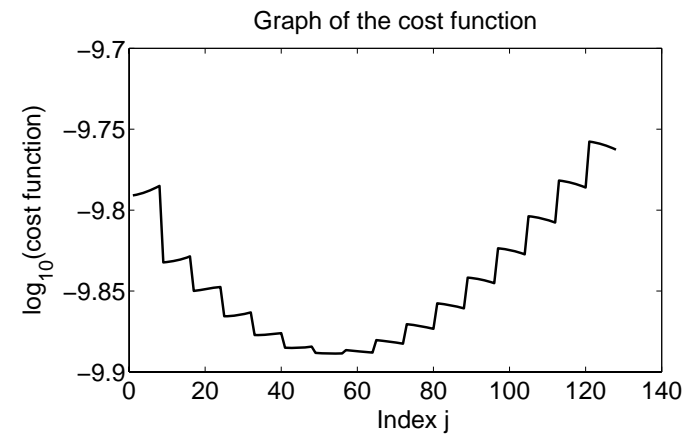

Figure 6: Cost function in $\log _{10}$ scale: graph of $j \mapsto \log _{10} \mathcal{J}^{\text {post }}\left(\varepsilon_{1, j} \mathcal{Y}^{\text {opt }}, \varepsilon_{2, j} \mathbf{1}\right)$.

and yields the optimal value $\varepsilon_{1}^{\text {opt }}=0.965$ and $\varepsilon_{2}^{\text {opt }}=0.045$. The probability density function $u \mapsto p_{U_{k}^{\text {post }}}\left(u ; \underline{\mathcal{Y}}^{\text {opt }}, \boldsymbol{\sigma}^{\text {opt }}\right)$ is estimated with the posterior model $\boldsymbol{U}^{\text {post }}\left(\underline{\mathcal{Y}}^{\text {opt }}, \boldsymbol{\sigma}^{\text {opt }}\right)=\boldsymbol{h}\left(\mathbb{V}^{\text {post }}\left(\underline{\mathcal{Y}}^{\text {opt }}, \boldsymbol{\sigma}^{\text {opt }}\right)\right)$. In order to validate the method, we compare $p_{U_{k}^{\text {post }}}$ with the experiments. Then an approximation $\widehat{p}_{U_{k}^{\exp }}\left(u ; \nu_{\exp }\right)$ of the probability density function $u \mapsto p_{U_{k}^{\exp }}(u)$ of the experimental random observation vector $U_{k}^{\text {exp }}$ is estimated with the experimental data $\left\{\boldsymbol{u}^{\exp , 1}, \ldots, \boldsymbol{u}^{\exp , \nu_{\exp }}\right\}$. Fig. 7 shows the comparison of $u \mapsto \log _{10} p_{U_{k}^{\text {post }}}\left(u ; \underline{\mathcal{Y}}^{\text {opt }}, \boldsymbol{\sigma}^{\text {opt }}\right)$ with $u \mapsto \log _{10} \widehat{p}_{U_{k}} \exp \left(u ; \nu_{\text {exp }}\right)$ and with $u \mapsto \log _{10} p_{U_{k}^{\text {prior }}}\left(u ; \mathcal{Y}^{\text {opt }}\right)$ for two observed DOF $k$ corresponding to the $x_{2}$ - 
displacement of nodes 19 and 27 located inside the face $x_{1}=0$. The probability density functions are estimated using the kernel density estimation method. The
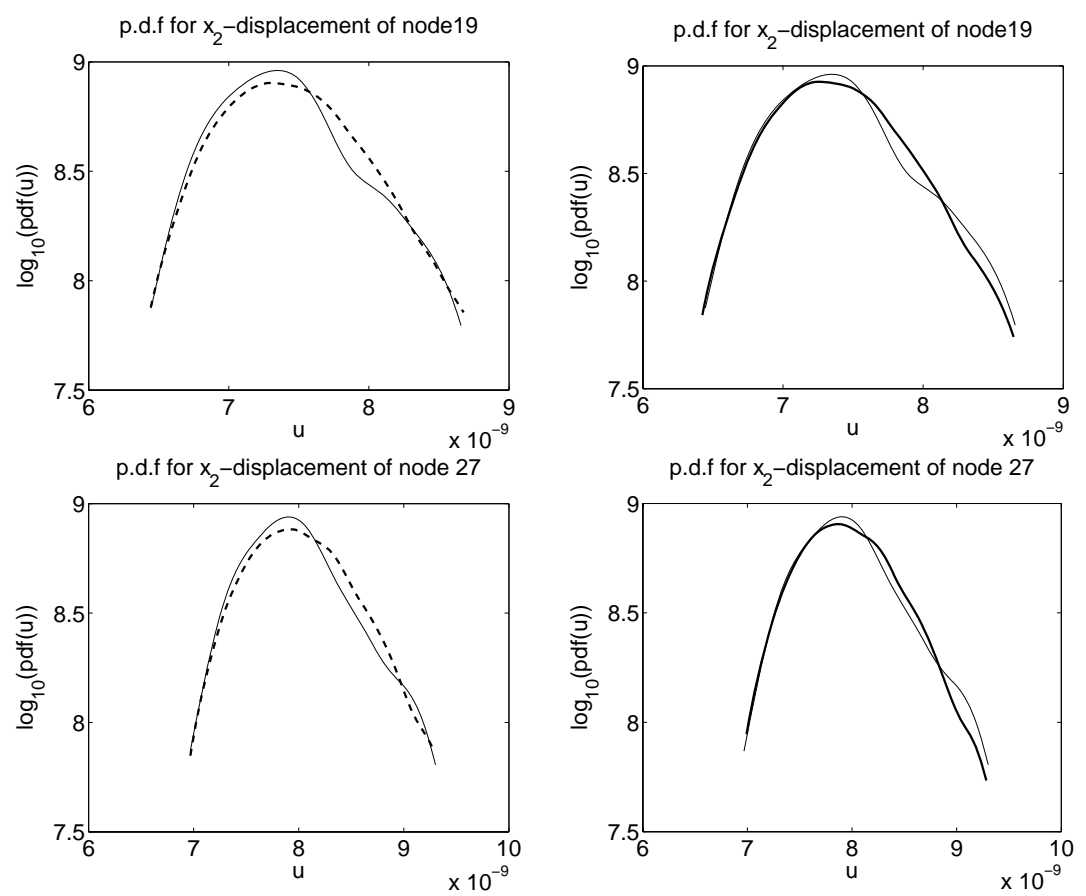

Figure 7: For observed DOF $k$ corresponding to the $x_{2}$-displacement of nodes 19 (top figures) and 27 (down figures), graphs of $u \mapsto \log _{10} \widehat{p}_{U_{k}} \exp \left(u ; \nu_{\exp }\right)$ (thin solid lines), $u \mapsto \log _{10} p_{U_{k}^{\text {prior }}}\left(u ; \mathcal{Y}^{\text {opt }}\right)$ (dashed thick lines), $u \mapsto \log _{10} p_{U_{k}^{\text {post }}}\left(u ; \underline{\mathcal{Y}}^{\text {opt }}, \boldsymbol{\sigma}^{\text {opt }}\right)$ (thick solid lines).

two figures on left show that the prior probability density functions are already a good approximation of the experimental probability density functions thanks to the identification with the experimental data of the optimal prior model which has been performed in Step 2. The two figures on right show that the posterior probability density functions are better approximations of the experimental probability density functions with respect to the prior probability density functions, even if the improvement stays small, but significant, in this case.

(9) Quality assessment of the posterior stochastic model. In Subsection (8) above, we have compared the probability density function $u \mapsto p_{U_{k}^{\mathrm{post}}}\left(u ; \underline{\mathcal{Y}}^{\mathrm{opt}}, \boldsymbol{\sigma}^{\mathrm{opt}}\right)$ with the reference solution represented by the probability density function $u \mapsto$ $p_{U_{k}^{\exp }}(u)$ of the experimental random observation vector $U_{k}^{\exp }$. The comparaisons shown in Fig. 7 are related to the observed DOF for which experimental data 
were available and have thus been used in the identification procedure. In order to give a quality assessment of the posterior stochastic model, we present a comparison of these quantities for DOF which have not been used to identify the posterior stochastic model. We then consider the two additional observed DOF $k^{\prime}$ corresponding to the $x_{2}$-displacement of the node 76 for which the coordinates are $(0.1667,0.500,0.8333)$ and of the node 170 for which the coordinates are $(0.500,0.500,0.1667)$. These two nodes are located inside the cube. Fig. 8 shows the comparison of $u \mapsto \log _{10} p_{U_{k^{\prime}}^{\text {post }}}\left(u ; \underline{\mathcal{Y}}^{\text {opt }}, \boldsymbol{\sigma}^{\text {opt }}\right)$ with $u \mapsto \log _{10} \widehat{p}_{U_{k^{\prime}}} \exp \left(u ; \nu_{\text {exp }}\right)$ and with $u \mapsto \log _{10} p_{U_{k^{\prime}}^{\text {prior }}}\left(u ; \mathcal{Y}^{\text {opt }}\right)$ The probability density functions are estimated using the kernel density estimation method. The two figures on right validate the quality of the posterior stochastic model for degrees of freedom which have not be used to identify it.
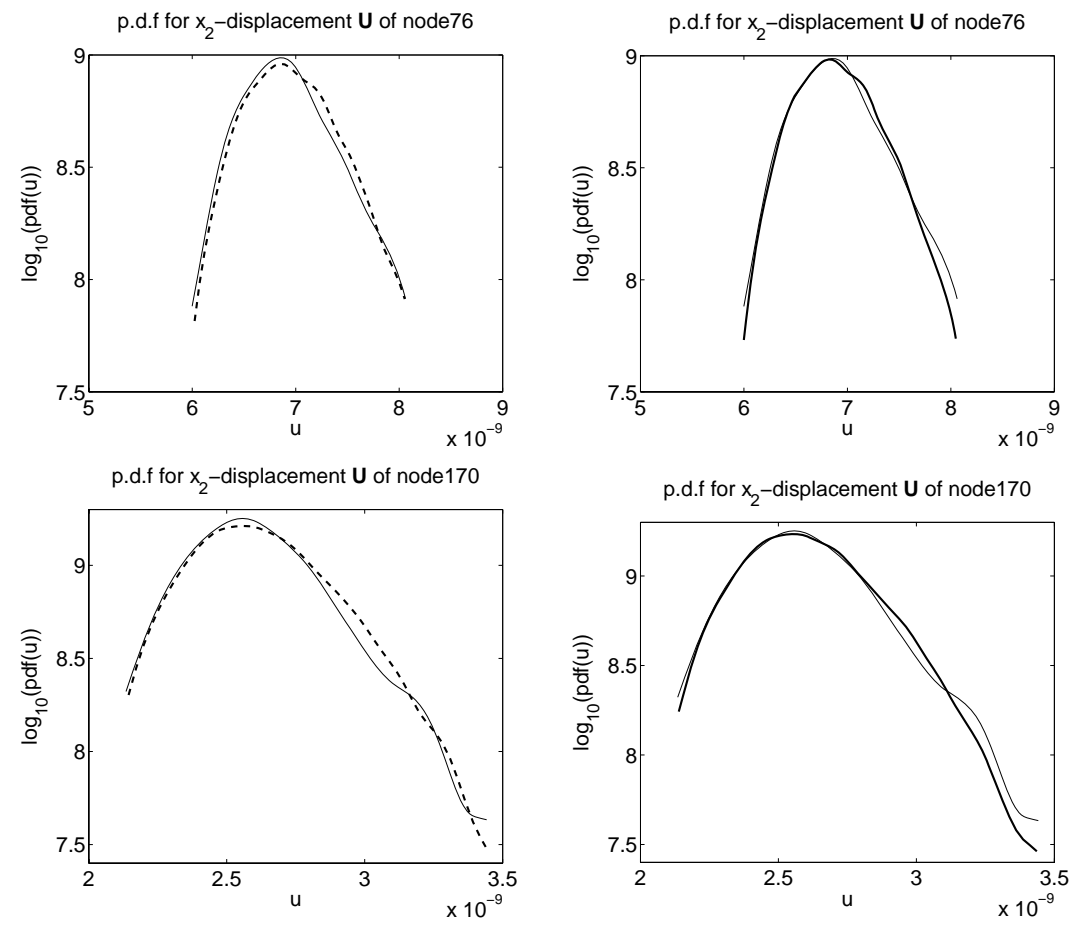

Figure 8: For DOF $k^{\prime}$ not used in the identification of the posterior stochastic model and corresponding to the $x_{2}$-displacement of nodes 76 (top figures) and 170 (down figures), graphs of $u \mapsto \log _{10} \widehat{p}_{U_{k^{\prime}}}^{\exp }\left(u ; \nu_{\exp }\right)$ (thin solid lines), $u \mapsto \log _{10} p_{U_{k^{\prime}}^{\text {prior }}}\left(u ; \mathcal{Y}^{\text {opt }}\right)$ (dashed thick lines), $u \mapsto \log _{10} p_{U_{k^{\prime}}^{\text {post }}}\left(u ; \underline{\mathcal{Y}}^{\text {opt }}, \boldsymbol{\sigma}^{\text {opt }}\right)$ (thick solid lines). 


\section{Conclusions}

An approach has been proposed to identify polynomial chaos expansion in high dimension of tensor-valued random field with partial experimental data through a stochastic boundary value problem. A complete methodology made up of five steps and associated algorithms have been proposed. The application presented shows the effective capability of the approach presented. With such a new methodology, the inverse identification and the convergence analysis of the polynomial chaos expansion with deterministic coefficients has been able to be done for tensorvalued random field which constitutes a novel result. Concerning the last step devoted to the construction and the inverse identification of the polynomial chaos expansion with random coefficients, a constructive approach based on the use of the log error function of the probability density function has been proposed. The asymptotic sampling Gaussian distribution constructed with the Fisher information matrix recently introduced could also be used as well as the Bayesian posteriors. Finally, if experimental data corresponding to direct field measurements (in all the domain) by image processing of the random field are available, then the identification of the polynomial chaos expansion in high dimension can directly be performed using Step 3 and Step 4.

\section{References}

[1] M. Arnst, R. Ghanem, C. Soize, Identification of Bayesian posteriors for coefficients of chaos expansion, Journal of Computational Physics, in press (2010), doi: 10.1016/j.jcp.2009.12.033.

[2] I. Babuska, F. Nobile, R. Tempone, A stochastic collocation method for elliptic partial differential equations with random input data, SIAM Journal on Numerical Analysis 45(3) (2007) 1005-1034.

[3] I. Babuska, R. Tempone, G.E. Zouraris, Solving elliptic boundary value problems with uncertain coefficients by the finite element method: the stochastic formulation, Computer Methods in Applied Mechanics and Engineering 194(12-16) (2005) 1251-1294.

[4] A.W. Bowman, A. Azzalini, Applied Smoothing Techniques for Data Analysis, Oxford University Press, 1997. 
[5] J.L. Beck, L.S. Katafygiotis, Updating models and their incertainties. I: Bayesian statistical framework, Journal of Engineering Mechanics, 124(4) (1998) 455-461 .

[6] S. Das, R. Ghanem, S. Finette, Polynomial chaos representation of spatiotemporal random field from experimental measurements, Journal of Computational Physics, 228 (2009) 8726-8751.

[7] S. Das, R. Ghanem, J. Spall, Asymptotic sampling distribution for polynomial chaos representation of data: A maximum-entropy and fisher information approach, SIAM Journal on Scientific Computing, 30(5) (2008) 2207-2234.

[8] B.J. Debusschere, H.N. Najm, P.P. Pebay, et al., Numerical challenges in the use of polynomial chaos representations for stochastic processes, SIAM Journal on Scientific Computing, 26(2) (2004) 698-719.

[9] C. Desceliers, R. Ghanem, C. Soize, Maximum likelihood estimation of stochastic chaos representations from experimental data, International Journal for Numerical Methods in Engineering, 66(6) (2006) 978-1001.

[10] C. Desceliers, C. Soize, R. Ghanem, Identification of chaos representations of elastic properties of random media using experimental vibration tests, Computational Mechanics, 39(6) (2007) 831-838.

[11] B. Ganapathysubramanian, N. Zabaras, Sparse grid collocation schemes for stochastic natural convection problems, Journal of Computational Physics, 225(1) (2007) 652-685.

[12] R. Ghanem, Ingredients for a general purpose stochastic finite elements formulation, Computer Methods in Applied Mechanics and Engineering 168(1-4) (1999) 19-34.

[13] R. Ghanem R., S. Dham, Stochastic finite element analysis for multiphase flow in heterogeneous porous media, Transp. Porous Media 32 (1998) 239-262.

[14] R. Ghanem, R. Doostan, Characterization of stochastic system parameters from experimental data: A bayesian inference approach, Journal of Computational Physics, 217(1) (2006) 63-81.

[15] R. Ghanem, D. Ghosh, Efficient characterization of the random eigenvalue problem in a polynomial chaos decomposition, International Journal for $\mathrm{Nu}$ merical Methods in Engineering 72(4) (2007) 486-504. 
[16] R. Ghanem, R. Doostan, J. Red-Horse, A probability construction of model validation, Computer Methods in Applied Mechanics and Engineering, Vol: 197, No: 29-32, pp. 2585-2595, 2008.

[17] R. Ghanem, S. Masri, M. Pellissetti et al, Identification and prediction of stochastic dynamical systems in a polynomial chaos basis, Computer Methods in Applied Mechanics and Engineering 194(12-16) (2005) 1641-1654.

[18] R. Ghanem, M. Pellissetti, Adaptive data refinement in the spectral stochastic finite element method, Comm. Numer. Methods Engrg. 18 (2002) 141-151.

[19] R. Ghanem, J. Red-Horse, Propagation of uncertainty in complex physical systems using a stochastic finite element approach, Phys. D 133 (1999) 137144.

[20] R. Ghanem, P.D. Spanos, Polynomial chaos in stochastic finite elements, Journal of Applied Mechanics - Transactions of teh ASME 57(1) (1990) 197202.

[21] R. Ghanem, P.D. Spanos, Stochastic Finite Elements: A spectral Approach (revised edition), Dover Publications, New York, 2003.

[22] D. Ghosh, C. Farhat, Strain and stress computation in stochastic finite element methods, International Journal for Numerical Methods in Engineering, 74(8) (2008) 1219-1239.

[23] D. Ghosh, R. Ghanem, Stochastic convergence acceleration through basis enrichment of polynomial chaos expansions, International Journal for Numerical Methods in Engineering, 73(2) (2008) 162-184.

[24] J. Guilleminot, C. Soize, D. Kondo, C. Benetruy, Theoretical framework and experimental procedure for modelling volume fraction stochastic fluctuations in fiber reinforced composites, International Journal of Solid and Structures 45(21) (2008) 5567-5583.

[25] E.T. Jaynes, Information theory and statistical mechanics, Physical Review 106 (4) (1957) 620-630 and 108 (2) (1957) 171-190.

[26] J.N. Kapur, H.K. Kesavan, Entropy Optimization Principles with Applications, Academic Press, San Diego, 1992. 
[27] O.M. Knio, O.P. Le Maitre, Uncertainty propagation in CFD using polynomial chaos decomposition, Fluid Dynamics Research 38(9) (2006) 616-640.

[28] O.P. Le Maitre, A Newton method for the resolution of steady stochastic Navier-Stokes equations, Computers and Fluids, 38(8) (2009) 1566-1579.

[29] O.P. Le Maitre, M. Reagan, H. Najm, R. Ghanem, O. Knio, A stochastic projection method for fluid flow. II. Random process, J. Comput. Phys. 181 (2002) 9-44.

[30] O.P. Le Maitre, H. Najm, R. Ghanem, O. Knio, Multi-resolution analysis of Wiener-type uncertainty propagation schemes, J. Comp. Phys., 197(2) (2004) 502-531.

[31] O.P. Le Maitre, O. Knio, H. Najm, R. Ghanem, Uncertainty propagation using Wiener-Haar expansions, J. Comp. Phys. 197 (2004) 28-57.

[32] D. Lucor, C.H. Su, G.E. Karniadakis, Generalized polynomial chaos and random oscillators, International Journal for Numerical Methods in Engineering, 60(3) (2004) 571-596.

[33] D. Lucor, J. Meyers, P. Sagaut, Sensitivity analysis of large-eddy simulations to subgrid-scale-model parametric uncertainty using polynomial chaos, Journal of Fluid Mechanics 585 (2007) 255-279.

[34] X. Ma, N. Zabaras, An efficient Bayesian inference approach to inverse problems based on an adaptive sparse grid collocation method, Inverse Problems, 25(3) (2009) Article Number: 035013.

[35] Y.M. Marzouk, H.N. Najm, L.A. Rahn, Stochastic spectral methods for efficient Bayesian solution of inverse problems, Journal of Computational Physics 224(2) (2007) 560-586.

[36] Y.M. Marzouk, H.N. Najm, Dimensionality reduction and polynomial chaos acceleration of Bayesian inference in inverse problems, Journal of Computational Physics, 228(6) (2009) 1862-1902.

[37] H.N. Najm, Uncertainty quantification and polynomial chaos techniques in computational fluid dynamics, Journal Review of Fluid Mechanics 41 (2009) 35-52. 
[38] A. Nouy, Generalized spectral decomposition method for solving stochastic finite element equations: Invariant subspace problem and dedicated algorithms, Computer Methods in Applied Mechanics and Engineering, 197(51-52) (2008) 4718-4736.

[39] A. Nouy, A. Clement, F. Schoefs, et al, An extended stochastic finite element method for solving stochastic partial differential equations on random domains, Computer Methods in Applied Mechanics and Engineering 197(51-52) (2008) 4663-4682.

[40] M. Paffrath, U. Wever, Adapted polynomial chaos expansion for failure detection, Journal of Computational Physics 226(1) (2007) 263-281.

[41] J.R. Red-Horse, A.S. Benjamin, A probabilistic approach to uncertainty quantification with limited information, Reliability Engineering and System Safety 85 (2004) 183190.

[42] C.P. Rupert, C.T. Miller, An analysis of polynomial chaos approximations for modeling single-fluid-phase flow in porous medium systems, Journal of Computational Physics 226(2) (2007) 2175-2205.

[43] S. Sakamoto, R. Ghanem, Polynomial chaos decomposition for the simulation of non-Gaussian nonstationary stochastic processes, Journal of Engineering Mechanics-ASCE 128(2) (2002) 190-201.

[44] G.I. Schueller (Ed.), A state-of-the-art report on computational stochastic mechanics, Probabilistic Engineering Mechanics 12(4) (1997) 197-313.

[45] G.I. Schueller, Computational stochastic mechanics - recent advances, Computers and Structures 79(22-25) (2001) 2225-2234.

[46] G.I. Schueller (Ed.), Computational methods in stochastic mechanics and reliability analysis, Computer Methods in Applied Mechanics and Engineering 194(12-16) (2005) 1251-1795.

[47] G.I. Schueller, On the treatment of uncertainties in structural mechanics and analysis, Computers and Structures 85(5) (2007) 235-243.

[48] R .J. Serfling, Approximation Theorems of Mathematical Statistics, John Wiley \& Sons, 1980. 
[49] C.E. Shannon, A mathematical theory of communication, Bell System Technology Journal 27 (1948) 379-423 and 623-659.

[50] C. Soize, Non Gaussian positive-definite matrix-valued random fields for elliptic stochastic partial differential operators, Computer Methods in Applied Mechanics and Engineering, 195 (2006), 26-64.

[51] C. Soize, Tensor-valued random fields for meso-scale stochastic model of anisotropic elastic microstructure and probabilistic analysis of representative volume element size, Probabilistic Engineering Mechanics 23 (2008) 307-323.

[52] C. Soize, Construction of probability distributions in high dimension using the maximum entropy principle. Applications to stochastic processes, random fields and random matrices, International Journal for Numerical Methods in Engineering 76(10) (2008) 1583-1611.

[53] C. Soize, Generalized probabilistic approach of uncertainties in computational dynamics using random matrices and polynomial chaos decompositions, International Journal for Numerical Methods in Engineering, 81(8) (2010) 939-970.

[54] C. Soize, C. Desceliers, Computational aspects for constructing realizations of polynomial chaos in high dimension with arbitrary measure, Journal of Computational Physics (submitted 31 August 2009).

[55] C. Soize, R. Ghanem, Physical systems with random uncertainties : Chaos representation with arbitrary probability measure, SIAM Journal on Scientific Computing 26(2) (2004) 395-410.

[56] C. Soize, R. Ghanem, Reduced chaos decomposition with random coefficients of vector-valued random variables and random fields, Computer Methods in Applied Mechanics and Engineering, 198(21-26) (2009), 1926-1934.

[57] J.C. Spall, Introduction to Stochastic Search and Optimization, John Wiley and Sons, Hoboken, New Jersey, 2003.

[58] P.D. Spanos, R. Ghanem, Stochastic finite element expansion for random media, Journal of Engineering Mechanics, ASCE 115(5) (1989) 1035-1053.

[59] G. Stefanou, A. Nouy, A. Clément, Identification of random shapes from images through polynomial chaos expansion of random level set functions, International Journal for Numerical Methods in Engineering 79(2) (2009) 127-155. 
[60] E. Walter, L. Pronzato, Identification of Parametric Models from Experimental Data, Springer, 1997.

[61] X.L. Wan, G.E. Karniadakis, An adaptive multi-element generalized polynomial chaos method for stochastic differential equations, Journal of Computational Physics, 209(2) (2005) 617-642.

[62] X.L. Wan, G.E. Karniadakis, Multi-element generalized polynomial chaos for arbitrary probability measures, SIAM Journal on Scientific Computing 28(3) (2006) 901-928.

[63] X.L. Wan, G.E. Karniadakis, Solving elliptic problems with non-Gaussian spatially-dependent random coefficients, Computer Methods in Applied Mechanics and Engineering, 198(21-26) (2009) 1985-1995

[64] J.B. Wang, N. Zabaras, A Bayesian inference approach to the inverse heat conduction problem, International Journal of Heat and Mass Transfer, 47(1718) (2004) 3927-3941.

[65] J.B. Wang, N. Zabaras, Hierarchical Bayesian models for inverse problems in heat conduction, Inverse Problems, 21(1) (2005) 183-206.

[66] D.B. Xiu, G.E. Karniadakis, Wiener-Askey polynomial chaos for stochastic differential equations, SIAM Journal on Scientific Computing 24(2) (2002) 619-644.

[67] D.B. Xiu, G.E. Karniadakis, Modeling uncertainty in steady state diffusion problems via generalized polynomial chaos Computer Methods in Applied Mechanics and Engineering, 191(43) (2002) 4927-4948.

[68] D.B. Xiu, G.E. Karniadakis, Modeling uncertainty in flow simulations via generalized polynomial chaos, Journal of Computational Physics, 187(1) (2003) 137-167.

[69] X.F. Xu, A multiscale stochastic finite element method on elliptic problems involving uncertainties, Computer Methods in Applied Mechanics and Engineering, 196(25-28) (2007) 2723-2736.

[70] N. Zabaras, B. Ganapathysubramanian, A scalable framework for the solution of stochastic inverse problems using a sparse grid collocation approach, Journal of Computational Physics, 227(9) (2008) 4697-4735. 